\title{
Una propuesta de tipología de sistemas de partidos para regímenes democráticos y autoritarios pluralistas. Su aplicación a los países del Magreb
}

\author{
A Proposal of Party Systems Typology for Democratic and Pluralist \\ Authoritarian Regimes. Its Application to the Maghreb Countries
}

Inmaculada Szmolka y Lucía G.-del-Moral

\section{Palabras clave}

Argelia

- Autoritarismo

pluralista

- Democracia

- Marruecos

- Regímenes políticos

- Sistemas de partidos

- Tipologías

- Túnez

\section{Key words}

Algeria

- Pluralist

Authoritarianism

- Democracy

- Morocco

- Political Regimes

- Party Systems

- Typologies

- Tunisia

\section{Resumen}

Desde el trabajo de Sartori, son escasas las tipologías de sistemas de partidos elaboradas, y las más recientes se centran en uno o dos criterios de clasificación, teniendo como referente las democracias. Este artículo propone una tipología de sistemas de partidos aplicable tanto en regímenes democráticos como en autoritarios pluralistas. Está basada en cuatro dimensiones: competencia, estabilidad del sistema de partidos, número y equilibrio entre partidos y polarización. Para verificar su validez, la tipología se aplica a tres regímenes políticos del Magreb: Túnez (democracia defectiva), Marruecos (autoritarismo cuasi competitivo) y Argelia (autoritarismo hegemónico). Su aplicación corrobora la validez de la tipología como forma de caracterizar los sistemas de partidos y permitir las investigaciones comparadas transnacionales.

\section{Abstract}

Since the pioneering work by Sartori, few typologies have been created for party systems and the most recent ones have focused on one or two classification criteria, using democracies as their reference. This article proposes a typology of party systems that can be applied to both democratic and pluralist authoritarian regimes. It is based on four dimensions: competition, party system stability, number and balance between parties and polarization. To verify its validity, the typology was applied to three political regimes of Maghreb: Tunisia (defective democracy) Morocco (quasi-competitive authoritarianism) and Algeria (hegemonic authoritarianism). Its application corroborates the validity of the typology as a possible way of characterizing party systems and allowing transnational research.

\section{Cómo citar}

Szmolka, Inmaculada y Moral, Lucía G.-del (2019). «Una propuesta de tipología de sistemas de partidos para regímenes democráticos y autoritarios pluralistas. Su aplicación a los países del Magreb». Revista Española de Investigaciones Sociológicas, 168: 93-110. (http://dx.doi.org/10.5477/ cis/reis.168.93)

La versión en inglés de este artículo puede consultarse en http://reis.cis.es

Inmaculada Szmolka: Universidad de Granada, Departamento de Ciencia Política y de la Administración | iszmolka@ugr.es Lucía G.-del-Moral: Universidad de Granada, Departamento de Ciencia Política y de la Administración | lugmm@correo.ugr.es 


\section{INTRODUCCIÓN1}

Las características de los sistemas de partidos tienen efectos considerablemente importantes en el funcionamiento de los sistemas políticos y la persistencia o cambio de los regímenes políticos. Por ello, la clasificación de los sistemas de partidos constituye un instrumento muy útil como punto de partida de la investigación comparada.

No obstante, la atención prestada al estudio de los sistemas de partidos en las últimas décadas es limitada (Wolinetz, 2004 y 2006; Bardi y Mair, 2008: 147; Golosov, 2011: 540). Así, son escasas las nuevas propuestas de categorización de los sistemas de partidos después de los trabajos pioneros de Duverger (1954), Blondel (1968), La Palombara y Weiner (1966) y, fundamentalmente, Sartori (1976). Las clasificaciones recientes se centran en una o dos dimensiones de los sistemas de partidos, como la institucionalización (Mainwaring y Scully, 1995), el número de partidos (Siaroff, 2000, 2003, 2006; Wolinetz, 2004 y 2006) y la polarización (Wolinetz, 2004 y 2006).

Por otra parte, hay que señalar que las tipologías de sistemas de partidos han sido pensadas para aplicarlas a las democracias. Por tanto, los sistemas de partidos de autoritarismos pluralistas o bien no han sido considerados o bien han sido reducidos a la categoría de sistemas de partido hegemónico (Sartori, 1976; Caramani, 2011: 327). Conforme al hecho de que la atención sobre los sistemas de partidos se ha centrado en las democracias, los casos de estudio se corresponden principalmente con países de Europa y de América ${ }^{2}$ y, en menor medida,

\footnotetext{
1 Este artículo ha sido realizado en el marco del proyecto de investigación financiado por la Junta de Andalucía «Persistencia del autoritarismo y procesos de cambio político en el Norte de África y Oriente Próximo: consecuencias sobre los regímenes políticos y el escenario internacional» (SEJ 2012-3118).

2 Blondel, 1968; Sartori, 1976; Daalder y Mair, 1983; Lijphart, 1994; Mair, 2002; Ware, 1996; Coppedge, 1998;
}

del África subsahariana 3 y Asia 4 . Por el contrario, no se ha elaborado ni aplicado ninguna clasificación de sistemas de partidos que tenga como referente el conjunto de países del Norte de África y Oriente Próximo.

Desde estas consideraciones, este artículo pretende responder a los siguientes interrogantes: ¿es posible una tipología de sistemas de partidos aplicable tanto a regímenes democráticos como a autoritarios pluralistas? ¿Qué parámetros deben tenerse en cuenta en la clasificación de los sistemas de partidos? ¿Los sistemas de partidos de la región del Norte de África y Oriente Próximo son susceptibles de ser categorizados bajo una tipología general de sistemas de partidos? Este artículo parte de la hipótesis de que una categorización general y multidimensional de sistemas de partidos es posible, además de deseable, si se quieren realizar comparaciones trasnacionales. Por ello, se propone una tipología general de sistemas de partidos basada en cuatro dimensiones: competencia, estabilidad del sistema de partidos, número y equilibrio entre partidos y polarización del sistema de partidos.

Para ilustrar la idoneidad de esta clasificación, esta se aplica a tres países del Magreb: Túnez (democracia defectiva), Marruecos (autoritarismo pluralista cuasi competitivo) y Argelia (autoritarismo pluralista hegemónico) (Szmolka, 2010 y 2017: 351). La selección de los casos se justifica por tres motivos: permite analizar sistemas de partidos de diferentes tipos de regímenes políticos, no existen clasificaciones de los sistemas de partidos en esta área geográfica y porque el conocimiento de las autoras sobre

Alcántara, del-Campo y Ramos, 2001; Jones, 2007; Dalton, 2008; Mainwaring, 2018.

3 Van-de-Walle y Butler, 1999; Bogaards, 2004; Lindberg, 2007; Erdmann y Basedau, 2008; Pelizzo y Nwokora, 2016.

4 Hicken y Martínez-Kuhonta, 2011; Croissant y Völkel, 2012. 
los tres países permite detectar problemas metodológicos de la tipología y errores de clasificación. Como unidades de análisis se han tenido en cuenta los sistemas de partidos resultantes de las elecciones: en Marruecos, de 2002, 2007, 2011 y 2016, celebradas bajo el reinado de Mohamed VI; en Argelia, de 1997, 2002, 2007, 2012 y 2017 tras la guerra civil; y en Túnez, de 2011 y 2014 , correspondientes a la nueva etapa democrática tras la caída del régimen de Ben Ali.

El artículo se estructura de la siguiente forma. En primer lugar, se conceptualizan los regímenes de autoritarismo pluralista y se destaca la ausencia de estudios sobre los sistemas de partidos de estos países. En segundo lugar, se presenta la propuesta teórica y metodológica para una tipología general y multidimensional de los sistemas de partidos. En tercer lugar, se analiza esta tipología en los tres casos seleccionados en función de cada una de las dimensiones clasificatorias. Y, por último, se concluye con los resultados obtenidos sobre la viabilidad de la propuesta, las dificultades encontradas y las sugerencias para la realización de futuras investigaciones sobre sistemas de partidos.

\section{LOS REGÍMENES AUTORITARIOS PLURALISTAS}

El objetivo de este artículo es proponer una clasificación general de sistemas de partidos que pueda aplicarse no solo en las democracias, sino también en aquellos regímenes autoritarios que cuentan con partidos que concurren periódicamente a procesos electorales generales. Estos últimos regímenes pueden denominarse como autoritarismos pluralistas (Szmolka, 2010). Otros autores se han referido a ellos como «autoritarismos competitivos» (Levitsky y Way, 2002; Diamond, 2002). No obstante, con este calificativo se hace hincapié en una propiedad de los sistemas políticos, la competencia política, que está limitada en los regímenes autoritarios. Así, el término de «pluralista» parece más idóneo para reflejar la posibilidad de enfrentamiento en el juego político entre varias opciones políticas.

En este sentido, mientras que en las democracias la única restricción que se establece al pluralismo y a la participación política es aquella que suponga una vulneración de los principios legales y constitucionales, en los regímenes autoritarios pluralistas alguna o algunas fuerzas políticas se pueden ver relegadas de los procesos políticos, siendo la competencia política limitada. Igualmente, el correcto funcionamiento del gobierno puede verse alterado por la falta de autonomía de las instituciones, la falta de equilibrio o contrapesos entre poderes o la presencia de actores influyentes sin responsabilidad política o por actores formales que centralizan el proceso institucional y político en detrimento de las instituciones representativas. Por otro lado, aunque se reconoce formalmente el Estado de derecho, en los regímenes autoritarios pluralistas se produce el recorte de derechos y libertades públicas cuando se considera en riesgo las bases del poder político.

En función del grado de competencia política, los regímenes autoritarios pluralistas pueden ser considerados como cuasi competitivos o hegemónicos. La diferencia principal entre ellos se encuentra en las mayores irregularidades de las elecciones, la posición ultradominante de un determinado partido o coalición en los procesos políticos y en la práctica imposibilidad de alternancia gubernamental por las urnas que se produce en los autoritarismos pluralistas hegemónicos (Szmolka, 2010).

A pesar de las diferencias que puedan existir entre las democracias y los autoritarismos pluralistas, lo cierto es que las funciones que los partidos cumplen en ambos regímenes son similares: agregación de in- 
tereses y demandas sociales, representación, reclutamiento de personal político, movilización, socialización, participación, formación de gobierno y elaboración de las políticas públicas (Hinnebush, 2017; Storm, 2017; Cavatorta y Storm, 2018).

Esto justifica la pertinencia y relevancia de estudiar los sistemas de partidos también en los regímenes autoritarios pluralistas. Sin embargo, solo Sartori (1976) ha realizado una clasificación de sistemas de partido en regímenes no democráticos, diferenciando entre sistemas de partido único y sistemas de partido hegemónico5. La categoría de sistemas de partido único no resulta pertinente desde un enfoque teórico basado en la existencia de al menos cierto pluralismo y competencia política. Por otro lado, la categoría de sistemas de partido hegemónico de Sartori, en la que un partido cuenta con el poder y el resto de fuerzas políticas autorizadas actúan como meros satélites de este sin que sea posible la alternancia, no agota los posibles casos que se pueden encontrar en la realidad actual, en donde la competencia por el poder puede ser mayor e, incluso, permitir la rotación del poder bajo los límites impuestos por el régimen. Asimismo, los sistemas de partidos en regímenes autoritarios pluralistas presentan también diferencias en cuanto a las divisiones ideológicas $u$ otros cleavages sociales relevantes, o en función de lo asentado que esté el sistema de partidos en el régimen político.

Por todo ello, a continuación se presenta una tipología general de sistemas de partidos con la pretensión de que pueda ser aplicada con independencia del tipo de régimen político considerado y que abarque las dimensiones más relevantes de los sistemas de partidos.

\footnotetext{
5 Caramani (2011: 319) reproduce la clasificación de Sartori para regímenes autoritarios en los mismos términos.
}

\section{Propuesta de tipología DE SISTEMAS DE PARTIDOS}

La tipología propuesta parte de la consideración del carácter multidimensional de los sistemas de partido (Gross y Sigelman, 1984: 463; Wolinetz, 2004 y 2006; Bardi y Mair, 2008: 149; Croissant y Völkel, 2016: 237). Por ello, la tipología contempla cuatro dimensiones clave en los sistemas de partidos: competencia, estabilidad del sistema de partidos, número y equilibrio entre los partidos y polarización (tabla 1).

\section{Competencia partidista}

Dado que la finalidad de este artículo es ofrecer una tipología aplicable tanto a regímenes democráticos como autoritarios, el primer criterio definitorio que se tiene en cuenta es la competencia partidista, entendida como la capacidad de los partidos para disputarse y acceder al poder. El grado de competencia se determina a partir de las siguientes variables:

1) Pluralismo político. Se estudia el reconocimiento legal de partidos y/o movimientos políticos, los límites formales o prácticos a la creación y actuación de los partidos, la representatividad de los partidos en función de los cleavages sociales relevantes (ideológicos, religiosos, étnicos, territoriales, etc.) y la posible exclusión de partidos del sistema político. El pluralismo político se analiza a través de las constituciones, leyes de partidos y prohibiciones o ilegalizaciones de partidos.

2) Integridad electoral. El objetivo es conocer si las elecciones son libres y limpias y si permiten parlamentos representativos y la formación de gobiernos democráticos. La integridad electoral se mide a través del Perceptions of Electoral Integrity Index (PEI), elaborado por la Universidad de Harvard y la Universidad de Sídney. Este índice posee una cobertura completa de países que celebran elecciones y abarca todas las fases del ciclo electoral. EI PEI considera la integridad 
electoral: muy alta, cuando es mayor de 70 puntos; alta, 69-60; moderada, 59-50; baja, 49-40; y muy baja, menos de 40.

3) Alternancia gubernamental. Se pretende constatar si se ha producido cambio en los partidos que lideran la formación del gobierno tras las elecciones o, al menos, si existe la posibilidad de que ocurra.

En función de estos criterios, se diferencian sistemas de partidos:

a) Competitivos. Se permite el libre juego político y el ejercicio de una oposición real al gobierno. La integridad de los procesos electorales es alta o muy alta, por lo que la posibilidad de alternancia gubernamental es real.

b) Cuasi competitivos. Existe reconocimiento legal de los partidos y en el sistema político participa un amplio espectro de fuerzas políticas con capacidad para disputarse el poder y estar representadas en las instituciones. Los partidos de oposición están presentes en las instituciones, pueden criticar al gobierno y proponer programas alternativos. No obstante, algún partido puede ser excluido por el régimen o no participa al considerar que no se dan las condiciones democráticas para ello. Por otro lado, las elecciones tienen lugar sin graves acusaciones de fraude, aunque se observan irregularidades que favorecen a los partidos oficialistas (integridad electoral moderada). La alternancia en el gobierno es posible de acuerdo a los resultados electorales.

c) Hegemónicos. La competencia se encuentra limitada por la posición hegemónica de un determinado partido, grupo o coalición en el sistema y los procesos políticos, por las trabas a la actividad de oposición o por graves irregularidades en los procesos electorales (integridad electoral baja o muy baja). De esta forma, se permite la interacción de los partidos en el sistema político, pero no la competencia real en el acceso al poder, ya que no existe posibilidad de alternancia gubernamental.

d) No pluralistas. La competencia se encuentra anulada por la ausencia misma de pluralismo y de la posibilidad de acceso al poder por medios electivos. No se admite la existencia de partidos o grupos políticos distintos a quienes ejercen el poder, por lo que no cabe hablar de sistemas de partidos.

\section{Estabilidad del sistema de partidos}

La segunda dimensión parte de la dicotomía planteada por Sartori (1976: 310) entre sistemas de partidos cristalizados y fluidos, en función de su desarrollo y continuidad en el tiempo. Por su parte, Mainwaring y Scully (1995) aplicaron el concepto de institucionalización a los sistemas de partidos, distinguiendo entre institucionalizados e incipientes 6 . Señalaron cuatro características de los sistemas de partidos institucionalizados: estabilidad en las reglas y en la naturaleza de la competición partidista, raíces estables en la sociedad, legitimidad de los partidos y de las elecciones y fortaleza de la organización de los partidos.

La institucionalización del sistema de partidos es, por tanto, un concepto multidimensional que Mainwaring y Scully miden a través de indicadores como la volatilidad electoral, la diferencia de votos entre las elecciones presidenciales y legislativas, los datos de encuesta e información cualitativa sobre el apego a los partidos, antigüedad de los partidos y evidencias cualitativas sobre la organización de los partidos. A partir de los criterios de Mainwaring y Scully se han ela-

6 La relevancia que se atribuye a la institucionalización del sistema de partidos procede de su relación con la consolidación democrática. No obstante, su análisis es también relevante en regímenes autoritarios pluralistas, pues un sistema de partidos institucionalizado puede influir en la resiliencia del autoritarismo (Hicken y Martínez-Kuhonta, 2011: 573). 
borado otras formas de medir el concepto de institucionalización más sistemáticas, destacando las de Jones (2007) y Croissant y Völkel (2012). Asimismo, Lindberg (2007) utiliza ocho indicadores para categorizar los sistemas de partidos como desestabilizados, fluidos o estables.

El análisis de un concepto multidimensional tan complejo como el de institucionalización excede los propósitos de este artículo de construir una clasificación básica de los sistemas de partidos. Además, varias de las condiciones de Mainwaring y Scully resultan menos determinantes. Así, es compatible un sistema de partidos institucionalizado con una baja confianza e identificación partidista. Por otro lado, la organización de los partidos remite a una consideración del partido como «unidad» y no como «sistema» (Randall y Svasand, 2002: 5).

De esta forma, la tipología propuesta se centra en los siguientes elementos relacionados con la continuidad del sistema de partidos:

1) Origen del sistema de partidos. Se data a través del año en el que se celebraron las primeras elecciones legislativas pluralistas del actual régimen político. Se establece un umbral de veinte años, período de tiempo en que lo habitual es que se hayan celebrado al menos cuatro elecciones, para diferenciar entre los sistemas de partidos con una trayectoria no reciente o dilatada en el tiempo.

2) Estabilidad o cambio en la oferta partidista. Por un lado, se utiliza como indicador el porcentaje de nuevos partidos que se presentan a las elecciones (nuevas formaciones, partidos fusionados o escindidos), teniendo en cuenta un porcentaje superior al $15 \%$ como un cambio relevante de la oferta partidista. Por otro lado, se aplica el índice de Pedersen de volatilidad electoral agregada7, asumiendo que, en un sistema de partidos estable, la volatilidad electoral debe ser baja (por debajo del 15\%) 8 o coyuntural (en un determinado proceso electoral).

3) Estabilidad o cambio de los partidos parlamentarios. De forma similar, se emplea el porcentaje de nuevos partidos parlamentarios y la volatilidad agregada en su dimensión parlamentaria.

En función de esto, se categorizan los sistemas de partidos como:

a) Estables. La gran mayoría de los partidos poseen una amplia y continua trayectoria en el sistema político, la oferta partidista es continua y la volatilidad es baja o coyuntural.

b) Fluidos. A pesar de que el sistema de partidos pueda tener un origen dilatado en el tiempo, son constantes las reconfiguraciones de la escena partidista y, como consecuencia, se producen reorientaciones del voto en cada convocatoria electoral y del sistema de partidos parlamentario.

c) No estructurados. El sistema de partidos es de desarrollo reciente. Entre los escasos procesos electorales celebrados se produce un elevado cambio de la oferta electoral y de los partidos con representación parlamentaria.

\section{Número y equilibrio entre partidos}

El formato numérico del sistema de partidos influye en aspectos como la orientación del voto, la participación electoral, la representación política, la formación y estabilidad del gobierno, las coaliciones políticas, la generación de consensos o el conflicto político (Dalton, 2008: 902; Ruiz y Otero, 2013: 125).

\footnotetext{
7 El índice de Pedersen (1979) se calcula a través de la suma de los porcentajes absolutos de votos o escaños de cada partido de una elección a otra divididos entre dos. 8 Ersson y Lane (1998).
} 
Asimismo, en contextos no democráticos, la división partidista puede constituir un instrumento en manos de los dirigentes autoritarios para controlar el poder.

Existen diferentes formas de cuantificar el número de partidos en el análisis empírico. En su estudio pionero, Duverger (1954) distinguió entre sistemas de partido único, bipartidistas y multipartidistas en relación con el número de partidos parlamentarios existentes. Por su parte, Blondel (1968) examinó el porcentaje de voto obtenido por los dos partidos principales y la distribución de escaños. Sobre esa base identificó sistemas bipartidistas, de dos partidos y medio, sistemas multipartidistas con un partido predominante y sistemas multipartidistas sin partido predominante. Posteriormente, Ware (1996: 239) consideró los partidos que superan el $3 \%$ de los escaños como criterio para determinar el número de partidos, situando el análisis de los sistemas de partidos en la esfera institucional. Sin embargo, cualquier umbral de exclusión resulta arbitrario (Golosov, 2010: 172), además de que no refleja la influencia de cada partido en el sistema (Siaroff, 2003: 268). En este sentido, para Sartori (1976), un partido "cuenta» en tanto que afecta a la mecánica y al funcionamiento del sistema por su potencial de coalición o chantaje. No obstante, este criterio resulta difícil de operacionalizar. De esta forma, han surgido fórmulas para contabilizar los partidos del sistema considerando el peso relativo de cada formación. El índice más utilizado es el Número Efectivo de Partidos Parlamentarios (NEPP) o Electorales (NEPE) de Laakso y Taagepera (1979) ${ }^{9}$.

Por último, la clasificación de Siaroff (2000: 69-72; 2003: 271) de sistemas de par-

\footnotetext{
9 EI NEP es una medida que ofrece el peso ponderado de cada partido electoral o parlamentario en base a su fuerza relativa (en votos o escaños). La fórmula es $\mathrm{N}=1 / \sum_{i=1}^{n} p_{i}^{2}$, donde $\mathrm{N}$ es el número de partidos con votos o escaños y $p_{i}^{2}$ es la proporción de votos/escaños de cada partido al cuadrado.
}

tidos resulta muy útil, ya que tiene en cuenta tanto el número de partidos como el equilibrio entre ellos, permitiendo diferenciar varias categorías de multipartidismos moderada o altamente fraccionados y la relevancia de los partidos mayoritarios. Siaroff utiliza como indicadores el número de partidos con al menos el 3\% de los escaños (P3\%S), la concentración parlamentaria de los dos partidos mayoritarios (2PSC), la ratio de escaños entre el primer y el segundo partido (SR1:2), la ratio entre el segundo y el tercer partido (SR2:3), y la media de NEPP en la que se sitúan los casos analizados. En función de ello, Siaroff (2000) clasifica a los sistemas de partidos como:

a) Bipartidistas. Los dos principales partidos concentran al menos el $95 \%$ de los escaños, existiendo un equilibrio entre ellos. Normalmente, un partido cuenta con mayoría absoluta. El número de partidos con más del $3 \%$ de escaños es de dos o tres y el NEPP de los casos analizados se sitúa en valores medios de 1,92 .

b) De dos partidos y medio. Los dos principales partidos ganan entre el $80-95 \%$ de los escaños, la ratio entre el primer y el segundo partido es inferior a 1,6 y la ratio entre el segundo y el tercero es de 1,8 o más. Es probable que uno de los dos principales partidos tenga mayoría absoluta y que los partidos que superan el $3 \%$ de los escaños sea de tres a cinco y normalmente de tres a cuatro (media de NEPP de 2,56).

c) De multipartidismo moderado con un partido dominante. De tres a cinco partidos superan el $3 \%$ de los escaños y la ratio entre el primer y el segundo partido es de 1,6 o más. Es posible que el partido principal tenga mayoría absoluta (media de NEPP de 2,95).

d) De multipartidismo moderado con dos partidos principales. De tres a cinco partidos superan el $3 \%$ de los escaños, la 
ratio entre el primer y el segundo partido es menor a 1,6 y la ratio entre el segundo y el tercer partido es 1,8 o superior (media de NEPP de 3,17 ).

e) De multipartidismo moderado con equilibrio entre los partidos. De tres a cinco partidos superan el $3 \%$ de escaños, la ratio entre el primer y el segundo partido es menor de 1,6 y la ratio entre el segundo y el tercer partido es menor de 1,8 (media de NEPP de 3,69).

f) De multipartidismo extremo con un partido dominante. Más de cinco partidos superan el $3 \%$ de escaños y la ratio entre los dos principales partidos es superior a 1,6 (media de NEPP de 3,96).

g) De multipartidismo extremo con dos partidos principales. Más de cinco partidos reciben el $3 \%$ de escaños, la ratio de los dos principales partidos es menor a 1,6 y la ratio entre el segundo y el tercer partido es de 1,8 o mayor. Aunque no es un rasgo definitorio, la concentración parlamentaria de los dos principales partidos se sitúa entre el 55 y el $75 \%$. Ninguno de los dos partidos tiene mayoría absoluta (media de NEPP de 4,41).

h) De multipartidismo extremo con equilibrio entre los partidos. Existen más de cinco partidos con el $3 \%$ de los votos, la ratio entre los dos principales partidos es menor de 1,6 y la ratio entre el segundo y el tercer partido es menor de 1,8. La concentración de los dos principales partidos suele ser menor del 60\% (media de NEPP de 5,56).

En la clasificación propuesta se utilizarán los indicadores y las categorías de Siaroff de la forma siguiente. En primer lugar, se determina el formato numérico del sistema de partidos calculando el NEPP. Dado que el NEPP que ofrece Siaroff para cada categoría de sistemas de partidos constituye una media de los casos que analiza, se emplea la conversión de Mainwaring y ScuIly (1995: 31-32) que otorga entre 1,8-2,4 a un sistema bipartidista; entre 2,5-2,9 a un sistema de partidos de dos y medio; entre 3-3,9 a un sistema de multipartidismo limitado y más de 4 puntos a un sistema de multipartidismo extremo. En el caso de los sistemas bipartidistas y de dos partidos y medio se tiene en cuenta también la concentración de escaños que es el criterio que identifica mejor estas categorías, utilizando los umbrales de Siaroff. En segundo lugar, se analiza el equilibrio del sistema de partidos, determinando si existe un escenario de partido dominante (SR1:2 $\geq 1,6)$ o no dominante $(<1,6)$ y, si no lo hay, comprobando si existe predominancia de dos partidos (SR $1: 3 \geq 1,8$ ) o equilibrio entre partidos $(<1,8)$.

\section{Polarización}

La relevancia del análisis de la polarización del sistema de partidos radica en su influencia sobre la competición partidista, el comportamiento electoral, la formación y estabilidad del gobierno o la adopción de acuerdos entre partidos, entre otros aspectos (Sani y Sartori, 1983: 337; Ocaña y Oñate, 1999: 237). Asimismo, la polarización puede influir en el éxito o fracaso de los procesos de democratización (Szmolka, 2017: 349).

Sartori (1976) fue el primero en señalar la polarización ideológica como elemento diferenciador de los sistemas de partidos. Identificó principalmente la polarización con los sistemas de multipartidismo extremo caracterizados por dinámicas de competición centrífuga y partidos antisistema. Otros autores han entendido también la polarización como la distancia ideológica entre partidos (Sigelman y Yough, 1978; Sani y Sartori, 1983; Hazan, 1997; Dalton, 2008). No obstante, la ideología no siempre describe la dimensión a lo largo de la cual los partidos (o los votantes) se dividen, sino que puede ser adecuado considerar otros cleavages (étnico, lingüístico, religioso, territorial, etc.), o una particular combinación de 
ellos (Stoll, 2008: 1445; Lauka, McCoy y Firat, 2018: 3).

Por otra parte, la polarización remite también a la posible agrupación de partidos en bloques. Así, Wolinetz $(2004,2006)$ tiene en cuenta en los sistemas de partidos el número de polos (unipolar, bipolar o multipolar) y la presencia/ausencia de alianzas permanentes o semipermanentes entre los partidos (clustering). Por su parte, Caramani (2011: 329 y 332) considera los sistemas bipolares como una categoría específica de los sistemas de partidos, determinados por la existencia de dos grandes coaliciones que suman alrededor del $80 \%$ de los votos y escaños, el equilibrio en su peso electoral y parlamentario (sobre el $40-50 \%$ del voto o de los escaños), la estabilidad de las coaliciones y su concurrencia en alianzas electorales, la alternancia en el gobierno entre las coaliciones y la formación de gobiernos de coalición.

Se han utilizado indicadores diversos para medir la polarización, como la ubicación de los partidos en el espectro ideológico izquierda-derecha o en familias políticas (Sartori, 1976; Sigelman y Yough, 1978; Gross y Sigelman, 1984) o las posiciones partidistas a través de sus programas políticos (Budge, Robertson y Hearl, 1987; Wildford, 2017). Estas formas de medición presentan el inconveniente de no tener en cuenta el peso relativo de cada partido (Ocaña y Oñate, 1999: 237). Por ello, se han elaborado índices para calcular la polarización, entre los que se encuentran los de Taylor y Herman (1971), Knutsen (1998) o Dalton (2008: 904).

En el caso de la clasificación propuesta, se mide la polarización a través de las siguientes variables:

1) Distancia entre los partidos parlamentarios. Si existen datos de ubicación de partidos, se utiliza el índice de polarización de Dalton. De forma adicional o sustitutiva, se tiene en cuenta la identificación de los parti- dos en familias políticas, prestando atención a la presencia de partidos en los extremos ideológicos (con un porcentaje de representación parlamentaria de más del 10\%) y/o de partidos antisistema. No obstante, este último indicador no resulta determinante, puesto que puede existir un escenario de polarización tanto en ausencia como en presencia de partidos antisistema. Así, en contextos autoritarios es prácticamente imposible su participación.

2) Alianzas partidistas estables o semiestables en relación con la concurrencia a las elecciones (con representación parlamentaria), la formación del gobierno (gobiernos de coalición o apoyos parlamentarios a su investidura) o procesos políticos relevantes (por ejemplo, reformas políticas).

3) Número de polos. En el caso de que exista un escenario de polarización partidista y una trayectoria de colaboración entre partidos, se determina el número de polos en los que los partidos se segmentan.

En consecuencia, se distingue:

a) Sistemas de partidos no polarizados unipolares. La amplia mayoría de partidos, entre ellos los más importantes, se sitúa en el centro o en posiciones cercanas al mismo.

b) Sistemas de partidos polarizados bipolares. Los partidos se agrupan en dos bloques equilibrados (porcentaje similar de escaños) y enfrentados en relación con la ideología o cualquier otro cleavage. Es habitual el establecimiento de coaliciones o alianzas partidistas (electorales, parlamentarias o gubernamentales) permanentes o semipermanentes.

c) Sistemas de partidos polarizados multipolares. Los partidos se agrupan en posiciones centrales y extremas (a ambos lados). Es habitual el establecimiento de coaliciones o alianzas partidistas (electorales, parlamentarias o gubernamentales) que pueden ser cambiantes. 
TABLA 1. Clasificación general y multidimensional de sistemas de partidos

\begin{tabular}{|c|c|c|c|c|}
\hline $\begin{array}{l}\text { Dimen- } \\
\text { siones }\end{array}$ & Variables & Indicador & Medición & Clasificación por categorías \\
\hline \multirow{6}{*}{ 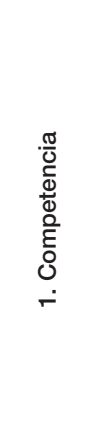 } & $\begin{array}{l}\text { Pluralismo } \\
\text { político }\end{array}$ & $\begin{array}{l}\text { Reconocimiento legal de } \\
\text { partidos }\end{array}$ & & \multirow{6}{*}{$\begin{array}{l}\text { Competitivo: pluralismo no limitado, } \\
\text { integridad electoral alta o muy alta, } \\
\text { alternancia gubernamental real o } \\
\text { factible } \\
\text { Cuasi competitivo: pluralismo } \\
\text { limitado, integridad electoral } \\
\text { moderada, alternancia gubernamental } \\
\text { real o factible } \\
\text { Hegemónico: pluralismo limitado, } \\
\text { integridad electoral baja o muy baja, } \\
\text { inexistencia de alternancia guberna- } \\
\text { mental } \\
\text { No competitivo: no pluralista }\end{array}$} \\
\hline & & $\begin{array}{l}\text { Restricciones legales a la } \\
\text { creación de partidos }\end{array}$ & $\begin{array}{c}\text { No pluralismo/ } \\
\text { Pluralismo limitado/ }\end{array}$ & \\
\hline & & & Pluralismo no limitado & \\
\hline & & $\begin{array}{l}\text { Partidos excluidos o autoex- } \\
\text { cluidos del sistema político }\end{array}$ & & \\
\hline & $\begin{array}{l}\text { Integridad } \\
\text { electoral }\end{array}$ & $\begin{array}{l}\text { Perceptions of Electoral } \\
\text { Integrity Index (PEI) }\end{array}$ & $\begin{array}{c}\text { Muy alta }(\geq 70) / \text { Alta }(69-60) / \\
\text { Moderada }(59-50) / \\
\text { Baja }(49-40) / \text { Muy baja }(<40)\end{array}$ & \\
\hline & $\begin{array}{l}\text { Alternancia } \\
\text { en el } \\
\text { gobierno }\end{array}$ & $\begin{array}{l}\text { Cambio de partidos en la } \\
\text { formación de gobierno }\end{array}$ & Sí/ No & \\
\hline \multirow{5}{*}{ 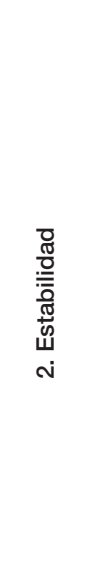 } & $\begin{array}{l}\text { Origen del } \\
\text { sistema de } \\
\text { partidos }\end{array}$ & $\begin{array}{l}\text { Año de las primeras } \\
\text { elecciones legislativas } \\
\text { (régimen actual) }\end{array}$ & $\begin{array}{l}\text { Reciente }(<20 \text { años }) / \\
\text { Dilatado }(>20)\end{array}$ & \multirow{5}{*}{$\begin{array}{l}\text { Estable: origen dilatado, oferta } \\
\text { electoral cristalizada, continuidad en la } \\
\text { composición parlamentaria y } \\
\text { volatilidad baja o coyuntural } \\
\text { Fluido: origen dilatado, oferta } \\
\text { partidista y composición parlamentaria } \\
\text { cambiante y alta volatilidad } \\
\text { Inestable: origen reciente, oferta } \\
\text { partidista y composición parlamentaria } \\
\text { cambiante y alta volatilidad }\end{array}$} \\
\hline & $\begin{array}{l}\text { Estabilidad } \\
\text { o cambio } \\
\text { en la oferta }\end{array}$ & $\begin{array}{l}\text { Porcentaje de nuevos parti- } \\
\text { dos sobre el total que se } \\
\text { presentan a las elecciones }\end{array}$ & $\begin{array}{l}\text { Oferta partidista electoral } \\
\text { cristalizada }(<15 \%) / \\
\text { fluida }(\geq 15 \%)\end{array}$ & \\
\hline & electoral & $\begin{array}{l}\text { Índice de Pedersen de } \\
\text { volatilidad electoral }\end{array}$ & $\begin{array}{c}\text { Volatilidad alta }(>15) / \\
\text { Moderada o baja }(<15) / \\
\text { Coyuntural (volatilidad }>15 \\
\text { en una sola elección) }\end{array}$ & \\
\hline & \multirow{2}{*}{$\begin{array}{l}\text { Estabilidad } \\
\text { o cambio } \\
\text { en los } \\
\text { partidos } \\
\text { parlamen- } \\
\text { tarios }\end{array}$} & $\begin{array}{l}\text { Porcentaje de nuevos } \\
\text { partidos parlamentarios }\end{array}$ & $\begin{array}{c}\text { Cambio parlamentario } \\
(>15 \%) / \text { Estabilidad }(<15 \%)\end{array}$ & \\
\hline & & $\begin{array}{l}\text { Índice de Pedersen de } \\
\text { volatilidad parlamentaria }\end{array}$ & $\begin{array}{c}\text { Volatilidad alta }(>15) / \\
\text { Moderada o baja }(<15) / \\
\text { Coyuntural (volatilidad }>15 \\
\text { en una sola legislatura) }\end{array}$ & \\
\hline \multirow{5}{*}{ 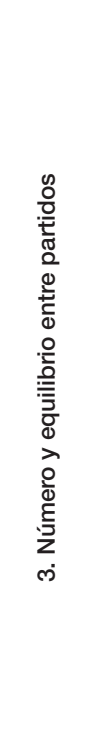 } & $\begin{array}{l}\text { Número de } \\
\text { partidos }\end{array}$ & $\begin{array}{l}\text { Número efectivo de } \\
\text { partidos parlamentarios }\end{array}$ & $\begin{array}{c}\text { Bipartidista }(1,8-2,4) ; \\
\text { de dos y medio }(2,5-2,9) ; \\
\text { multipartidismo moderado } \\
\text { (entre 3-3,9); } \\
\text { multipartidismo extremo }(>4)\end{array}$ & \multirow{5}{*}{$\begin{array}{l}\text { Bipartidista: NEPP } 1,8-2,4 \\
\text { (P3\%S=2-3); } 2 \text { PSC }>95 \% \text {. } \\
\text { De dos partidos y medio: NEPP } \\
2,5-2,9 ; 2 P S C=80-95 \% \text {; SR } 1: 2<1,6 ; \\
\text { SR2:3 } \geq 1,8 \text {. } \\
\text { Multipartidismo moderado con un } \\
\text { partido dominante: NEPP } 3-3,9 \\
\text { (P3\%S=3-5); SR1:2 } \geq 1,6 \text {. } \\
\text { Multipartidismo moderado con dos } \\
\text { partidos principales: NEPP } 3-3,9 \\
\text { (P3\%S=3-5); SR1:2<1,6; SR2:3 } \geq 1,8 \\
\text { Multipartidismo moderado con } \\
\text { equilibrio entre partidos: NEPP } 3-3,9 \\
\text { (P3\%S=3-5); SR1:2<1,6; SR2:3 }<1,8 \\
\text { Multipartidismo extremo con un } \\
\text { partido dominante: NEPP }>4 \text { (P3\%S } \\
>5 \text { ); SR1:2 } \geq 1,6 \\
\text { Multipartidismo extremo con dos } \\
\text { partidos principales: NEPP }>4 ; \\
\text { SR1:2<1,6; SR2:3 } \geq 1,8 \\
\text { Multipartidismo extremo con } \\
\text { equilibrio entre partidos: NEPP }>4 ; \\
\text { SR1:2<1,6; SR2:3 }<1,8\end{array}$} \\
\hline & & $\begin{array}{l}\text { Concentración } \\
\text { parlamentaria }\end{array}$ & 2PSC (>95\%; 80-95\%) & \\
\hline & $\begin{array}{l}\text { Predomi- } \\
\text { nancia/ } \\
\text { Equilibrio } \\
\text { entre } \\
\text { partidos }\end{array}$ & Dominancia de un partido & $\mathrm{SR} 1: 2 \geq 1,6$ & \\
\hline & & Dominancia de dos partidos & SR $1: 2<1,6$ y SR2:3 $\geq 1,8$ & \\
\hline & & Equilibrio entre partidos & SR $1: 2<1,6$ y SR2:3 $<1,8$ & \\
\hline
\end{tabular}


TABLA 1. Clasificación general y multidimensional de sistemas de partidos. (Continuación)

\begin{tabular}{|c|c|c|c|c|}
\hline $\begin{array}{l}\text { Dimen- } \\
\text { siones }\end{array}$ & Variables & Indicador & Medición & Clasificación por categorías \\
\hline \multirow{5}{*}{$\begin{array}{l}: \frac{}{U} \\
\frac{0}{\mathbb{N}} \\
\frac{N}{2} \\
\frac{\pi}{0} \\
0 \\
\dot{0}\end{array}$} & \multirow{3}{*}{$\begin{array}{l}\text { Distancia } \\
\text { entre los } \\
\text { partidos }\end{array}$} & $\begin{array}{l}\text { Índice de polarización de } \\
\text { Dalton }\end{array}$ & $\begin{array}{l}\text { Polarizado/ } \\
\text { No polarizado }\end{array}$ & \multirow{5}{*}{$\begin{array}{l}\text { No polarizado unipolar } \\
\text { Polarizado bipolar } \\
\text { Polarizado multipolar }\end{array}$} \\
\hline & & $\begin{array}{l}\text { Partidos parlamentarios en } \\
\text { los extremos ideológicos } \\
\text { (con más del } 10 \% \text { escaños) }\end{array}$ & & \\
\hline & & $\begin{array}{l}\text { Existencia o no de partidos } \\
\text { antisistema }\end{array}$ & & \\
\hline & $\begin{array}{l}\text { Coaliciones } \\
\text { de partidos }\end{array}$ & $\begin{array}{l}\text { Formación o no de } \\
\text { coaliciones electorales (con } \\
\text { representación), guberna- } \\
\text { mentales o políticas }\end{array}$ & $\begin{array}{l}\text { No existentes/ } \\
\text { Estables/ } \\
\text { Coyunturales }\end{array}$ & \\
\hline & Polos & $\begin{array}{l}\text { Número de polos en los } \\
\text { que se agrupan los partidos }\end{array}$ & Unipolar/Bipolar/Multipolar & \\
\hline
\end{tabular}

Fuente: Elaboración propia.

\section{Clasificación y Caracterización DE LOS SISTEMAS DE PARTIDOS de Marruecos, Argelia y Túnez}

\section{Competencia partidista}

La percepción de amplio pluralismo en el Magreb se desprende de los datos sobre el número de partidos que concurrieron a las últimas elecciones legislativas en cada país: 27 partidos se presentaron a los comicios de 2016 en Marruecos, 69 en Argelia en 2017 (más 163 listas independientes) y hasta 890 en Túnez en 2014 (más 151 coaliciones y 459 listas independientes).

No obstante, el pluralismo no es absoluto en los tres casos. La transición democrática en Túnez tras la caída del régimen de Ben Ali en 2011 permitió la libertad de creación de partidos con la única exclusión de aquellos que atenten contra la identidad o integridad del Estado. Por el contrario, pese a un amplio abanico de fuerzas políticas que cubre el espectro ideológico derechaizquierda, en Marruecos y Argelia se prohíbe la creación de partidos sobre una base religiosa, lingüística, racial o regional. Conforme a ello, varios partidos o movimientos no han sido autorizados o han sido disueltos (véase la tabla 2). Pese a ello, es posible identificar partidos con líderes y apoyo social bereberes: en Marruecos, el Movimiento Popular, y en Argelia, el Frente de Fuerzas Socialistas y la Agrupación por la Cultura y la Democracia. Igualmente, hay que mencionar partidos de referencia islamista que fueron legalizados y que cuentan actualmente con representación parlamentaria: el Partido de la Justicia y del Desarrollo (PJD) en Marruecos, el Movimiento de la Sociedad por la Paz (MSP), Islah y Ennahda, en Argelia, y Ennahda y Hizb ut-Tahir en Túnez.

Por otro lado, pluralismo no es sinónimo de competencia. Además de las restricciones a la creación de partidos, los procesos electorales de Marruecos y Argelia sufren de irregularidades. Según el PEI, durante el período 2012-2018, las elecciones se celebraron con un alto grado de integridad electoral en Túnez, moderado en Marruecos y bajo en Argelia.

Por último, la alternancia gubernamental ha sido posible en Marruecos y Túnez en dos ocasiones, pero no en Argelia. En Marruecos, en 1998, el partido opositor Unión Socialista de Fuerzas Populares (USFP) formó gobierno con otras seis fuerzas políticas y, en 2012, el partido islamista PJD lideró una coalición gubernamental con otros tres par- 
TABLA 2. Competencia partidista

\begin{tabular}{|c|c|c|c|c|}
\hline Variable & Indicador & Marruecos & Argelia & Túnez \\
\hline \multirow{3}{*}{$\begin{array}{l}\text { Pluralismo } \\
\text { político }\end{array}$} & $\begin{array}{l}\text { Reconocimiento } \\
\text { legal partidos }\end{array}$ & $\begin{array}{l}\text { Ley Orgánica 29-11, } \\
23 \text { de octubre de } 2011\end{array}$ & $\begin{array}{l}\text { Ley Orgánica 4-12, } 15 \text { de } \\
\text { enero de } 2012\end{array}$ & $\begin{array}{l}\text { Decreto Ley } 2011-87, \\
24 \text { de septiembre de } 2011\end{array}$ \\
\hline & $\begin{array}{l}\text { Restricciones } \\
\text { legales }\end{array}$ & $\begin{array}{l}\text { Partidos de base religiosa, } \\
\text { lingüística, étnica o regional }\end{array}$ & $\begin{array}{l}\text { Partidos de base } \\
\text { religiosa, lingüística, } \\
\text { racial, sexual, corporativa } \\
\text { o regional }\end{array}$ & No \\
\hline & $\begin{array}{l}\text { Partidos/ } \\
\text { movimientos } \\
\text { prohibidos, } \\
\text { no autorizados } \\
\text { o autoexcluidos }\end{array}$ & $\begin{array}{l}\text { Bereber: Partido Democráti- } \\
\text { co Amazigh Islamistas: } \\
\text { Partido al-Umma; Al-Badil } \\
\text { al-Hadari; Al-Adl wa al-Ihsan } \\
\text { Extrema izquierda: Annahj } \\
\text { Addimocrati }\end{array}$ & $\begin{array}{l}\text { Islamistas: Frente Islámico } \\
\text { de Salvación; Movimiento } \\
\text { por la Democracia; Wafa'; } \\
\text { Hizb ut-Tahir } \\
\text { Izquierda: Partido Árabe } \\
\text { Socialista Ba'ath }\end{array}$ & - \\
\hline $\begin{array}{l}\text { Integridad } \\
\text { electoral }\end{array}$ & PEI & Moderada (56) & Ваја (43) & Alta (67) \\
\hline $\begin{array}{l}\text { Alternancia } \\
\text { gubernamental }\end{array}$ & $\begin{array}{l}\text { Cambio de } \\
\text { partidos en la } \\
\text { formación de } \\
\text { gobierno }\end{array}$ & $\begin{array}{l}\text { 1998: Gobierno Yussufi } \\
\text { (USFP en coalición) } \\
\text { 2011: Gobierno Benkiran } \\
\text { (PJD en coalición) }\end{array}$ & No & $\begin{array}{l}\text { 2011: Gobierno Ghannuchi } \\
\text { (Ennahda en coalición) } \\
\text { 2014: Gobierno Chahed } \\
\text { (Nidaa Tounes en coalición) }\end{array}$ \\
\hline Clasificación & & Cuasi competitivo & Hegemónico & Competitivo \\
\hline
\end{tabular}

Fuente: Elaboración propia.

tidos. No obstante, en ambas ocasiones se produjo la «cohabitación» de partidos de la oposición con partidos oficialistas. En Túnez, la alternancia gubernamental se materializó como consecuencia de la transición democrática. Tras las elecciones a la Asamblea Constituyente de 2011, el partido ganador, Ennahda, formó gobierno junto con otros tres partidos. La segunda alternancia gubernamental tuvo lugar en 2014, cuando el movimiento de unidad nacional Nidaa Tounes lideró un gobierno de coalición con Ennahda y dos partidos minoritarios. Por el contrario, en Argelia no se han producido cambios en el gobierno tras la guerra civil, en manos de la coalición formada por el Frente de Liberación Nacional (FLN), la Agrupación Nacional Democrática (RND) y, hasta poco antes de las elecciones de 2017, el MSP.

En definitiva, el análisis realizado lleva a caracterizar el sistema de partidos como competitivo en Túnez, cuasi competitivo en Marruecos y hegemónico en Argelia.

\section{Estabilidad del sistema de partidos}

El origen del multipartidismo es distinto en los tres casos de estudio. A diferencia de los sistemas de partido único de los regímenes poscoloniales de Argelia (FLN) y Túnez (Partido Neodestur), en Marruecos se instauró el multipartidismo tras su independencia en 1956, teniendo lugar las primeras elecciones legislativas en 1962. La participación de otros partidos en el sistema político tunecino y argelino no se produjo hasta 1983 y 1989 , respectivamente. No obstante, la guerra civil abierta en Argelia en 1991 provocó un vacío institucional hasta la celebración de elecciones pluralistas presidenciales en 1995 y legislativas en 1997. Y, en Túnez, el proceso de transición democrática como consecuencia de la Primavera Árabe permitió la convocatoria de las primeras elecciones competitivas en octubre de 2011. En resumen, los actuales sistemas de partidos cuentan con una trayectoria parlamentaria reciente de 7 años en Túnez y dilatada de 56 años en Marruecos y de 21 en Argelia. 
TABLA 3. Estabilidad del sistema de partidos

\begin{tabular}{|c|c|c|c|c|}
\hline Variable & Indicador & Marruecos & Argelia & Túnez \\
\hline $\begin{array}{l}\text { Origen del sistema de } \\
\text { partidos }\end{array}$ & $\begin{array}{c}\text { Año primeras elecciones } \\
\text { legislativas }\end{array}$ & $\begin{array}{c}1962 \\
\text { Dilatado }\end{array}$ & $\begin{array}{c}1997 \\
\text { Dilatado }\end{array}$ & $\begin{array}{c}2011 \\
\text { Reciente }\end{array}$ \\
\hline \multirow{10}{*}{$\begin{array}{l}\text { Estabilidad o cambio en la } \\
\text { oferta electoral }\end{array}$} & \multirow{5}{*}{$\begin{array}{l}\text { Nuevos partidos } \\
\text { electorales (\%) }\end{array}$} & 16,6 (2016-2011) & $37,7(2017-2012)$ & \multirow[t]{4}{*}{$94,4(2014-2011$} \\
\hline & & $18,9(2011-2007)$ & $47,7(2012-2007)$ & \\
\hline & & 22,0 (2007-2002) & 4,2 (2007-2002) & \\
\hline & & & $17,4(2002-1997)$ & \\
\hline & & Oferta fluida & Oferta fluida & Oferta fluida \\
\hline & \multirow[t]{5}{*}{ Volatilidad electoral } & 15,7 (2016-2011) & $53,9(2017-2012)$ & \multirow[t]{4}{*}{76,4 (2014-2011 } \\
\hline & & 26,8 (2011-2007) & 43,3 (2012-2007) & \\
\hline & & 17,7 (2007-2002) & $41,0(2007-2002)$ & \\
\hline & & & $58,6(2002-1997)$ & \\
\hline & & Alta & Alta & Alta \\
\hline \multirow{10}{*}{$\begin{array}{l}\text { Estabilidad o cambio en los } \\
\text { partidos parlamentarios }\end{array}$} & \multirow{5}{*}{$\begin{array}{l}\text { Nuevos partidos } \\
\text { parlamentarios (\%) }\end{array}$} & 0 (2016-2011) & $34,4(2017-2012)$ & \multirow[t]{4}{*}{$60,0(2014-2011$} \\
\hline & & 33,3 (2011-2007) & $38,5(2012-2007)$ & \\
\hline & & $26,1(2007-2002)$ & $68,2(2007-2002)$ & \\
\hline & & & $44,4(2002-1997)$ & \\
\hline & & De cambio a estabilidad & Cambio & Cambio \\
\hline & \multirow[t]{5}{*}{ Volatilidad parlamentaria } & $19,2(2016-2011)$ & $38,6(2017-2012)$ & \multirow[t]{4}{*}{60,5 (2014-2011 } \\
\hline & & $26,0(2011-2007)$ & $33,7(2012-2007)$ & \\
\hline & & 21,6 (2007-2002) & $39,9(2007-2002)$ & \\
\hline & & & $58,6(2002-1997)$ & \\
\hline & & Alta & Alta & Alta \\
\hline Clasificación & & Fluido & Fluido & No estructurado \\
\hline
\end{tabular}

Fuente: Elaboración propia.

Por otro lado, la oferta electoral ha sido muy cambiante en Argelia y en Túnez, como se desprende de la variación del número de partidos que concurren a las elecciones y de la elevada volatilidad electoral. En Túnez, este hecho es consecuente con la transición democrática que posibilitó la creación de nuevos partidos, no solo ante la celebración de las elecciones fundacionales de ese año, sino también ante las de 2014. Asimismo, la Primavera Árabe supuso un incremento de nuevos partidos en Argelia, aunque la alta volatilidad electoral es un elemento característico del sistema de partidos argelino en todo el período. Pese a ello, estos cambios no han afectado la hegemonía de los dos partidos mayoritarios (FLN y RND) en Argelia.
Por lo que respecta a Marruecos, a pesar de contar con una trayectoria partidista más amplia, la alta volatilidad electoral y los cambios en la correlación de fuerzas parlamentarias siguen siendo una característica destacable. No obstante, en 2016 no entró ningún nuevo partido en el Parlamento, aunque desaparecieron seis respecto a la anterior legislatura (tabla 3).

Por todo ello, se ha caracterizado a los sistemas de partidos marroquí y argelino como fluidos, y al tunecino, como no estructurado.

\section{Número y equilibrio entre los partidos}

Los tres países de estudio comparten un sistema electoral proporcional de resto mayor 
TABLA 4. Número y equilibrio entre los partidos

\begin{tabular}{|c|c|c|c|c|c|c|c|c|c|c|c|}
\hline & \multicolumn{4}{|c|}{ Marruecos } & \multicolumn{5}{|c|}{ Argelia } & \multicolumn{2}{|c|}{ Túnez } \\
\hline & 2016 & 2011 & 2007 & 2002 & 2017 & 2012 & 2007 & 2002 & 1997 & 2014 & 2011 \\
\hline P3\%S & $8 / 12$ & $8 / 18$ & $10 / 23$ & $11 / 22$ & $7 / 34$ & $5 / 27$ & $7 / 23$ & $5 / 10$ & $6 / 10$ & $5 / 15$ & $5 / 19$ \\
\hline NEPP* & 5,0 & 6,6 & 10,4 & 10,1 & 4,8 & $\begin{array}{l}3,8 \\
4,1\end{array}$ & $\begin{array}{l}4,8 \\
5,5\end{array}$ & 2,8 & 3,9 & 3,6 & 4,3 \\
\hline 2PSC & 57,5 & 42,3 & 40,0 & 30,1 & 56,9 & 59,7 & 50,6 & 63,2 & 58,9 & 71,4 & 54,4 \\
\hline SR1:2 & 1,2 & 1,8 & 1,1 & 1,0 & 1,6 & 3,1 & 2,2 & 4,2 & 2,2 & 1,2 & 3,1 \\
\hline SR2:3 & 2,2 & 1,1 & 1,1 & 1,1 & 3,0 & 1,4 & 1,2 & 1,1 & 1,1 & 4,3 & 1,1 \\
\hline Clasificación & \multicolumn{4}{|c|}{$\begin{array}{c}\text { Multipartidismo extremo } \\
\text { con predominancia de dos } \\
\text { partidos }\end{array}$} & \multicolumn{4}{|c|}{$\begin{array}{c}\text { Multipartidismo extremo con } \\
\text { predominancia de dos } \\
\text { partidos }\end{array}$} & \multicolumn{3}{|c|}{$\begin{array}{c}\text { Multipartidismo moderado } \\
\text { con predominancia de dos } \\
\text { partidos }\end{array}$} \\
\hline
\end{tabular}

* Los candidatos independientes no han sido considerados en el cálculo del NEPP. En Argelia representan el 6,1\% en 2017, el $8,5 \%$ en 2007 , el 7,7\% en 2002 y el 2,9\% en 1997 . Y, en Túnez, el 1,4\% en las elecciones de 2014 y el $3,7 \%$ en 2011.

Fuente: Elaboración propia.

(cociente Hare) en circunscripciones plurinominales en las que se presentan listas cerradas y bloqueadas. Sin embargo, la fragmentación parlamentaria es mayor en Marruecos y Argelia que en Túnez.

Así, los sistemas actuales de partidos marroquí y argelino pueden categorizarse como de multipartidismo extremo con dos principales partidos. En ambos casos, la fragmentación parlamentaria es alta, arrojando el NEPP un valor cercano a 5 partidos. No obstante, en Marruecos, la fragmentación ha disminuido considerablemente desde que alcanzase su cuota más alta en la legislatura 2007-2011 (NEPP 10,4 partidos). Por otro lado, la ausencia de mayorías absolutas, la alta concentración de escaños entre las dos primeras fuerzas parlamentarias y la ratio elevada entre el segundo y el tercer partido en ambos países nos revela la supremacía de los dos partidos mayoritarios (tabla 4). Sin embargo, mientras que en Marruecos el segundo partido, el Partido de la Autenticidad y la Modernidad (PAM), constituye el principal partido de la oposición, en Argelia, la RND forma parte de la coalición de gobierno desde 1997 (Szmolka, 2018).

Por su parte, Túnez presenta actualmente un sistema de multipartidismo moderado con predominancia de dos partidos. Pese a ello, hasta 15 partidos se encuentran representados en el Parlamento, aunque solo 5 superan el $3 \%$ de los escaños. Nidaa Tounes y Ennahda concentran el $71,4 \%$ de los escaños y se encuentran muy separados del tercer partido clasificado. A pesar de sus diferencias ideológicas, ambos partidos participan en la coalición de gobierno.

\section{Polarización}

Dada la ausencia de datos sobre la ubicación ideológica de los partidos, no se ha podido calcular el índice de polarización de Dalton. Por ello, se han utilizado como indicadores alternativos la existencia de partidos parlamentarios en los extremos ideológicos y de partidos antisistema. Como se observa en la tabla 5, no existen partidos extremistas con representación parlamentaria ni partidos antisistema en los tres países. En Marruecos y Argelia esto responde a las características autoritarias de sus respectivos regímenes políticos, que obligan a los partidos a moderar su discurso y programas políticos, incluso a los partidos que pudieran ser más críticos, como los islamistas y los de izquierda. Tampoco en Túnez la existencia de un partido islamista como Ennahda polariza la escena 
TABLA 5. Polarización

\begin{tabular}{|c|c|c|c|c|}
\hline Variable & Indicador & Marruecos & Argelia & Túnez \\
\hline \multirow{2}{*}{$\begin{array}{l}\text { Distancia entre } \\
\text { partidos }\end{array}$} & $\begin{array}{l}\text { Partidos parlamentarios } \\
\text { en los extremos }\end{array}$ & No & No & No \\
\hline & Partidos antisistema & No & No & No \\
\hline \multirow[b]{2}{*}{ Coaliciones } & $\begin{array}{l}\text { Electorales (obteniendo } \\
\text { representación) }\end{array}$ & No & $\begin{array}{l}\text { 2017: MSP-FC } \\
\text { y Ennahda-FJD } \\
\text { 2012: AW (MSP, } \\
\text { Ennahda, Islah) }\end{array}$ & Frente Popular \\
\hline & Gubernamentales & $\begin{array}{l}2018 \text { y } 2017 \text { : } \\
\text { PJD, RNI, MP, USFP, } \\
\text { PPS, UC } \\
\text { 2013: } \\
\text { PJD, RNI, MP, PPS } \\
\text { 2012: } \\
\text { PJD, PI, MP, PPS } \\
\text { 2007: } \\
\text { PI, RNI, USFP, PPS } \\
\text { 2002 y 2004: } \\
\text { USFP, RNI, MP, MNP, } \\
\text { UC }\end{array}$ & $\begin{array}{l}\text { 2017: } \\
\text { FLN, RND, TAJ, MPA } \\
\text { 2017-2012: } \\
\text { FLN, RND } \\
\text { 1997-2012: } \\
\text { FLN, RND, MSP }\end{array}$ & $\begin{array}{l}\text { 2016: } \\
\text { Nidaa Tounes, Ennahda, } \\
\text { Afek Tounes, UPL } \\
\text { (fusionado con NT), Via } \\
\text { Democrática y Social, } \\
\text { Al-Yumhuri y al-Massar } \\
\text { (fuera en 2018) } \\
\text { 2015: } \\
\text { Nidaa Tounes, Ennahda, } \\
\text { Afek Tounes, UPL } \\
\text { 2011: Ennahda, } \\
\text { CPR, Ettakatol y PR }\end{array}$ \\
\hline Polos & & Uno & Uno & Uno \\
\hline Clasificación & & Unipolar no polarizado & Unipolar no polarizado & Unipolar no polarizado \\
\hline
\end{tabular}

Fuente: Elaboración propia.

partidista religiosa, dada su moderación desde sus orígenes y su participación en el gobierno desde 2011.

Por otro lado, la formación de coaliciones no determina la existencia de polarización. Por lo que respecta a las coaliciones electorales, dos obtuvieron representación en Argelia en 2017, ambas de carácter islamista. No obstante, la mayoría absoluta del FLN y de la RND impide la bipolarización en Argelia. En Túnez, la única coalición electoral destacable es la del Frente Popular, formada por nueve partidos, pero solo representa el 5\% de los escaños. En cuanto a las coaliciones de gobierno, en los tres países no se trata de coaliciones alternantes, sino que se caracterizan por ser sobredimensionadas, participando más partidos de los necesarios, sobre todo, en Túnez. Asimismo, son coaliciones ideológica y políticamente heterogéneas en las que cohabitan partidos islamistas con partidos oficialistas y, en Marruecos y Túnez, también de izquierda moderada.
En conclusión, los tres sistemas de partidos se caracterizan por ser no polarizados unipolares.

\section{Conclusiones}

Este artículo ha intentado ofrecer una nueva tipología de sistemas de partidos ante las escasas o limitadas clasificaciones realizadas en este ámbito tras el clásico trabajo de Sartori. La principal aportación de la tipología propuesta es que puede ser aplicada tanto en regímenes democráticos como en autoritarios pluralistas. Además, contempla las principales dimensiones de los sistemas de partidos: competencia partidista, estabilidad del sistema de partidos, número y equilibrio entre los partidos y polarización. Por tanto, la tipología permite las investigaciones comparadas trasnacionales sobre sistemas de partidos, así como una caracterización completa de los mismos. 
TABLA 6. Clasificación multidimensional de los sistemas de partidos

\begin{tabular}{lcccc}
\hline & Competencia & Estabilidad & $\begin{array}{c}\text { Número y equilibrio } \\
\text { entre partidos }\end{array}$ & Polarización \\
\hline Marruecos & Cuasi competitivo & Fluido & $\begin{array}{c}\text { Multipartidismo extremo con } \\
\text { dos partidos principales }\end{array}$ & Unipolar no polarizado \\
Argelia & Hegemónico & Fluido & $\begin{array}{c}\text { Multipartidismo extremo con } \\
\text { dos partidos principales }\end{array}$ & Unipolar no polarizado \\
Túnez & Competitivo & No estructurado & $\begin{array}{c}\text { Multipartidismo moderado } \\
\text { con dos partidos principales }\end{array}$ & Unipolar no polarizado \\
\hline
\end{tabular}

Fuente: Elaboración propia.

Para comprobar su validez, la tipología se ha aplicado a tres países del Magreb, lo que nos permite analizar sistemas de partidos en tres regímenes políticos distintos sobre los que no existen clasificaciones previas. El análisis de nuestros casos de estudio nos ha llevado a caracterizar los sistemas de partidos de la siguiente forma: Marruecos, sistema de partidos cuasi competitivo, fluido, de multipartidismo extremo con predominancia de dos partidos y no polarizado; Argelia, sistema de partidos hegemónico, fluido, de multipartidismo extremo con predominancia de dos partidos y no polarizado, y Túnez, sistema de partidos competitivo, no estructurado, de multipartidismo moderado con predominancia de dos partidos y no polarizado.

La aplicación de la tipología se vio dificultada por la falta de datos oficiales sobre el registro oficial de partidos y datos electorales incompletos, por lo que se usaron fuentes complementarias como ElectionGuide. Asimismo, el cálculo del índice de polarización de Dalton no fue posible al no existir datos de ubicación de partidos. Como alternativa, se recurrió a la clasificación de los partidos en familias ideológicas para determinar la existencia de fuerzas políticas en los extremos ideológicos. Por otro lado, trabajar por períodos representa cierta dificultad, ya que los índices pueden variar en cada uno de ellos, dificultando la caracterización de cada dimisión. No obstante, solo se encontró variaciones en uno de los indicadores de la di- mensión de estabilidad del sistema de partidos aplicado a Marruecos, en la que se reflejó una tendencia de cambio a estabilidad en los partidos parlamentarios.

Por tanto, la tipología ha mostrado su utilidad como instrumento para clasificar de forma general los sistemas de partidos, por lo que se considera que puede constituir un punto de partida válido para las investigaciones comparadas. No obstante, sería conveniente testar la tipología en otras áreas geográficas para comprobar su validez en regímenes políticos de diferente naturaleza.

\section{Bibliografía}

Alcántara, Manuel; Campo, Esther del y Ramos, María Luisa (2001). «La naturaleza de los sistemas de partidos políticos y su configuración en el marco de los sistemas democráticos en América Latina». Justicia Electoral, 15: 57-83.

Bardi, Luciano y Mair, Peter (2008). «The Parameters of Party Systems». Party Politics, 14(2): 147-162.

Blondel, Jean (1968). «Party Systems and Patterns of Government in Western Democracies». Canadian Journal of Political Science, 1(2): 180-203.

Bogaards, Matthijs (2004). «Counting Parties and Identifying Dominant Party Systems in Africa». European Journal of Political Research, 43(2): 173-197.

Budge, lan; Robertson, David y Hearl, Derek (eds.) (1987). Ideology, Strategy and Party Change: Spatial Analysis of Postwar Election Programs in 19 Democracies. Cambridge: Cambridge University Press. 
Caramani, Daniel (2011). Comparative Politics. Oxford: Oxford University Press.

Cavatorta, Francesco y Storm, Lise (2018). Political Parties in the Arab World. Continuity and Change. Edinburgh: Edinburgh University Press.

Coppedge, Michael (1998). "The Dynamic Diversity of Latin American Party Systems». Party Politics, 4(4): 547-568.

Croissant, Aurel y Völkel, Philip (2012). «Party Systems Types and Party System Institutionalization: Comparing New Democracies in East and Southest Asia». Party Politics, 18(2): 235-265.

Daalder, Hans y Mair, Peter (1983). Western European Party Systems. Beverly Hills, California/ London: Sage Publications.

Dalton, Russell (2008). «The Quantity and the Quality of Party Systems. Party System Polarization, Its Measurement, and Its Consequences». Comparative Political Studies, 41(7): 899-920.

Diamond, Larry (2002). «Elections Without Democracy. Thinking About Hybrid Regimes". Journal of Democracy, 13(2): 21-35.

Duverger, Maurice (1954). Political Parties: Their Organization and Activity in the Modern State. London: Methuen.

Erdmann, Gero y Basedau, Matthias (2008). «Political Parties in Africa: Problems of Categorising and Explaining Party Systems». Journal of Contemporary African Studies, 26(3): 241-258.

Ersson, Svante y Lane, Jan-Erik (1998). «Electoral Instability and Party System Change in Western Europe». En: Pennings, P. y Lane, J.-E. (eds.). Comparing Party System Change. London: Routledge.

Golosov, Gregorii (2010). «The Effective Number of Parties. A New Approach». Party Politics, 16(2): 171-192.

Golosov, Gregorii (2011). «Party System Classification. A Methodological Inquiry». Party Politics, 17(5): 539-560.

Gross, Donald y Sigelman, Lee (1984). «Party Systems: A Multidimensional Approach». Comparative Politics, 16(4): 463-479.

Hazan, Reuven (1997). Centre Parties. Polarization and Competition in European Parliamentary Democracies. London: Pinter.

Hicken, Allen y Martínez-Kuhonta, Eric (2011). «Shadows from the Past: Party System Institutionalization in Asia». Comparative Political Studies, 44(5): 572-597.
Hinnebush, Raymond (2017). «Political Parties in MENA: Their Functions and Development». British Journal of Middle Eastern Studies, 44(2): 159-175.

Jones, Mark P. (2007). «Political Parties and Party Systems in Latin America». Paper presented at the Symposium Prospects for Democracy in Latin America. University of North Texas, Denton, Texas.

Knutsen, Oddbjorn (1998). «Expert Judgments of the Left-Right Location of Political Parties: A Comparative Longitudinal Study». West European Politics, 21(2): 63-94.

La Palombara, Joseph y Weiner, Myron (1966). Political Parties and Political Development. Princeton: Princeton University Press.

Laakso, Marku y Taagepera, Reinn (1979). «"Effective" Number of Parties: A Measure with Application to West Europe». Comparative Political Studies, 12(1): 3-27.

Lauka, Alban; McCoy, Jennifer y Firat, Rengin (2018). «Mass Partisan Polarization: Measuring a Relational Concept». American Behavioral Scientist, 62(1): 107-126.

Levitsky, Steven y Way, Lucan A. (2002). «Elections Without Democracy. The Rise of Competitive Authoritarianism". Journal of Democracy, 13(2): 51-65.

Lijphart, Arend (1994). Electoral Systems and Party Systems: A Study of Twenty-Seven Democracies, 1945-1990. Toronto: Oxford University Press.

Lindberg, Staffam (2007). «Institutionalization of Party Systems? Stability and Fluidity among Legislative Parties in Africa's Democracies». Government and Opposition, 42(2): 215-241.

Mainwaring, Scott (2018). Party Systems in Latin America. Cambridge: Cambridge University Press.

Mainwaring, Scott y Scully, Timothy (1995). «Party Systems in Latin America». En: Mainwaring, S. y Scully, T. (eds.). Building Democratic Institutions: Party-systems in Latin America. Stanford, California: Stanford University Press.

Mair, Peter (2002). "Comparing Party Systems». En: LeDuc, L., Niemi, R. y Norris, P. (eds.). Comparing Democracies 2: New Challenges in the Study of Elections and Voting. Thousand Oaks, California: Sage.

Ocaña, Francisco y Oñate, Pablo (1999). Análisis de datos electorales. Madrid: CIS.

Pedersen, Mogens (1979). «The Dynamics of European Party Systems: Changing Patterns of Elec- 
toral Volatility". European Journal of Political Research, 7(1): 1-26.

Pelizzo, Riccardo y Nwokora, Zim (2016). «Bridging the Divide: Measuring Party System Change and Classifying Party Systems». Politics and Policy, 44(6): 1017-1052.

Randall, Vickey y Svasand, Lars (2002). «Party Institutionalization in New Democracies». Party Politics, 8(1): 5-29.

Ruiz, Leticia y Otero, Patricia (2013). Indicadores de partidos y sistemas de partidos. Madrid: CIS.

Sani, Giacomo y Sartori, Giovani (1983). «Polarization, Fragmentation and Competition in Western Democracies». En: Daalder, H. y Mair, P. (eds.). Western European Party Systems. Beverly Hills, California/London: Sage.

Sartori, Giovanni (1976). Parties and Party Systems: A Framework for Analysis. Cambridge: Cambridge University Press.

Siaroff, Alan (2000). Comparative European Party Systems: An Analysis of Parliamentary Elections Since 1945. Oxford: Taylor and Francis.

Siaroff, Alan (2003). «Two-And-A-Half-Party Systems and the Comparative Role of the "Half"». Party Politics, 9(3): 267-90.

Siaroff, Alan (2006). «A Typology of Contemporary Party Systems». Paper presentado al XX Congreso Mundial de la International Political Science Association. Fukouka, Japón. 9-13 de julio de 2006. Disponible en: http://paperroom.ipsa. org/papers/paper_5213.pdf, acceso el 8 de mayo de 2018.

Sigelman, Lee y Yough, Sing (1978). «Left-Right Polarization in National Party Systems: A CrossNational Analysis». Comparative Political Studies, 11(3): 355-379.

Stoll, Heather (2008). «Social Cleavages and the Number of Parties: How the Measures you Choose Affect the Answers you Get». Comparative Political Studies, 41: 1439-1465.

Storm, Lise (2017). «Parties and Party System Change». En: Szmolka, I. (ed.). Political Change in the Middle East and North Africa: After the
Arab Spring. Edinburgh: Edinburgh University Press.

Szmolka, Inmaculada (2010). «Los regímenes políticos híbridos: democracias y autoritarismos con adjetivos. Su conceptualización, categorización y operacionalización dentro de la tipología de regímenes políticos". Revista de Estudios Políticos, 147: 103-135.

Szmolka, Inmaculada (2011). «Democracias y autoritarismos con adjetivos: la clasificación de los países árabes dentro de una tipología general de regímenes políticos". Revista Española de Ciencia Política, 26: 11-62.

Szmolka, Inmaculada (2017). "Successful and Failed Transitions to Democracy». En: Szmolka, I. (ed.). Political Change in the Middle East and North Africa: After the Arab Spring. Edinburgh: Edinburgh University Press.

Szmolka, Inmaculada (2018). «A Real Electoral Duel between the PJD and the PAM? Analysing Constituency-level Competitiveness in the 2016 Moroccan Elections». British Journal of Middle Eastern Studies.

Taylor, Michael y Herman, Valentine (1971). «Party Systems and Government Stability». American Political Science Review, 65(1): 28-37.

Walle, Nicolas, van de y Butler, Kimberly (1999). «Political Parties and Party Systems in Africa's Illiberal Democracies». Cambridge Review of International Studies, 13(1): 14-28.

Ware, Alan (1996). Partidos políticos y sistemas de partidos. Madrid: Istmo.

Wildford, Alan (2017). «Polarization, Number of Parties, and Voter Turnout: Explaining Turnout in 26 OECD Countries». Social Science Quarterly, 98(5): 1391-1405.

Wolinetz, Steven (2004). "Classifying Party Systems: Where Have All the Typologies Gone?». Annual Meeting of the Canadian Political Science Association. Winnipeg, Manitoba.

Wolinetz, Steven (2006). «Party Systems and Party System Types». En: Katz, R. y Crotty, W. (eds.). Handbook on Political Parties. London: Sage. 


\title{
A Proposal of Party Systems Typology for Democratic and Pluralist Authoritarian Regimes. Its Application to Maghreb Countries
}

\author{
Una propuesta de tipología de sistemas de partidos para regímenes \\ democráticos y autoritarios pluralistas. Su aplicación a los países del Magreb
}

Inmaculada Szmolka and Lucía G.-del-Moral

\section{Key words}

Algeria

- Pluralist

Authoritarianism

- Democracy

- Morocco

- Political Regimes

- Party Systems

- Typologies

- Tunisia

\section{Palabras clave}

Argelia

- Autoritarismo

pluralista

- Democracia

- Marruecos

- Regímenes políticos

- Sistemas de partidos

- Tipologías

- Túnez

\begin{abstract}
Since the pioneering work by Sartori, few typologies have been created for party systems and the most recent ones have focused on one or two classification criteria, using democracies as their reference. This article proposes a typology of party systems that can be applied to both democratic and pluralist authoritarian regimes. It is based on four dimensions: competition, party system stability, number and balance between parties and polarization. To verify its validity, the typology was applied to three political regimes of Maghreb: Tunisia (defective democracy) Morocco (quasi-competitive authoritarianism) and Algeria (hegemonic authoritarianism). Its application corroborates the validity of the typology as a possible way of characterizing party systems and allowing transnational research.
\end{abstract}

\section{Resumen}

Desde el trabajo de Sartori, son escasas las tipologías de sistemas de partidos elaboradas, y las más recientes se centran en uno o dos criterios de clasificación, teniendo como referente las democracias. Este artículo propone una tipología de sistemas de partidos aplicable tanto en regímenes democráticos como en autoritarios pluralistas. Está basada en cuatro dimensiones: competencia, estabilidad del sistema de partidos, número y equilibrio entre partidos y polarización. Para verificar su validez, la tipología se aplica a tres regímenes políticos del Magreb: Túnez (democracia defectiva), Marruecos (autoritarismo cuasi competitivo) y Argelia (autoritarismo hegemónico). Su aplicación corrobora la validez de la tipología como forma de caracterizar los sistemas de partidos y permitir las investigaciones comparadas transnacionales.

\section{Citation}

Szmolka, Inmaculada and Moral, Lucía G.-del- (2019). "A Proposal of Party Systems Typology for Democratic and Pluralist Authoritarian Regimes. Its Application to Maghreb Countries". Revista Española de Investigaciones Sociológicas, 168: 93-110. (http://dx.doi.org/10.5477/cis/reis.168.93)

Inmaculada Szmolka: Universidad de Granada, Departamento de Ciencia Política y de la Administración | iszmolka@ugr.es Lucía G.-del-Moral: Universidad de Granada, Departamento de Ciencia Política y de la Administración | lugmm@correo.ugr.es 


\section{INTRODUCTION 1}

The characteristics of the party systems have major effects on the functioning of political systems and the persistence or change of political regimes. Therefore, the classification of these party systems is a very useful instrument to initiate comparative research.

Over recent decades however, little attention has been paid to studies of party systems (Wolinetz 2004 and 2006; Bardi and Mair 2008: 147; Golosov 2011: 540). So, few new proposals have appeared for the categorization of party systems since the pioneering works by Duverger (1954), Blondel (1968), La Palombara and Weiner (1966) and, above all, Sartori (1976). Recent classifications have focused on one or two dimensions of party systems such as institutionalization (Mainwaring y Scully, 1995), the number of parties (Siaroff, 2000, 2003, 2006; Wolinetz, 2004 and 2006) and polarization (Wolinetz, 2004 and 2006).

On the other hand, it should be noted that the typologies of the party systems have mainly been created for application to democracies. So, party systems of pluralist authoritarianisms have either not been considered or have been reduced to a hegemonic party system category (Sartori 1976; Caramani 2011: 327). Since attention to party systems has focused on democracies, case studies correspond mainly to countries in Europe and America ${ }^{2}$ or, to a lesser degree,

\footnotetext{
1 This article has been created within the framework of the research project financed by the Junta of Andalusia: "Persistencia del autoritarismo y procesos de cambio político en el Norte de África y Oriente Próximo: consecuencias sobre los regímenes políticos y el escenario internacional" (SEJ 2012-3118).

2 Blondel, 1968; Sartori, 1976; Daalder and Mair, 1983; Lijphart, 1994; Mair, 2002; Ware, 1996; Coppedge, 1998; Alcántara, del-Campo and Ramos, 2001; Jones, 2007; Dalton, 2008; Mainwaring, 2018.
}

Sub-Saharan Africa ${ }^{3}$ and Asia ${ }^{4}$. No classification of party systems has been either created or applied to the set of countries making up North Africa or the Middle East.

Given these considerations, this article attempts to respond to the following questions: Is it possible to have a typology of party systems that applies both to democratic and pluralist authoritarian regimes?; What criteria should be considered in the classification of party systems?; Can the party systems of the Northern Africa and Middle Eastern areas be categorized under a general party system typology? This article is based on the hypothesis that a general and multi-dimensional categorization of party systems is possible, as well as desirable, in order to make transnational comparisons. To do so, a general typology of party systems has been proposed based on four dimensions: competition, stability of the party system, number and balance between parties and polarization of the party system.

To illustrate the suitability of this classification system, it has been applied to three countries of Maghreb: Tunisia (defective democracy), Morocco (quasi-competitive pluralist authoritarianism) and Algeria (hegemonic pluralist authoritarianism) (Szmolka, 2010 y 2017: 351). The selection of these country cases may be justified by three main reasons: it permits the analysis of distinct types of political regime party systems; no classifications exist for the party systems in this geographic area; and the authors' knowledge of the three countries permits the detection of methodological problems with the typology and classification errors. The party systems resulting

\footnotetext{
3 Van-de-Walle and Butler, 1999; Bogaards, 2004; Lindberg, 2007; Erdmann and Basedau, 2008; Pelizzo and Nwokora, 2016.

4 Hicken and Martínez-Kuhonta, 2011; Croissant and Völkel, 2012.
} 
from the following elections were considered as units of analysis: in Morocco, from 2002, 2007, 2011 and 2016, held under the reign of Mohammed VI; in Algeria, from 1997, 2002, 2007, 2012 and 2017 following the civil war; and, in Tunisia, from 2011 and 2014, corresponding to the new democratic era following the fall of the Ben Ali regime.

This article is structured as follows. First, the pluralist authoritarianism regimes are conceptualized and the absence of studies regarding the party systems of these countries is highlighted. Second, a theoretical and methodological proposal is presented for a general and multi-dimensional typology of the party systems. Third, this typology is analyzed for the three selected cases, based on each of the classification dimensions. And finally, conclusions are presented based on the results obtained, regarding the viability of the proposal, the difficulties encountered and suggestions for future studies on party systems.

\section{Pluralist authoritarian Regimes}

The objective of this article is to propose a general classification of the party systems that may be applied not only to democracies, but also to authoritarian regimes having parties that periodically participate in the general election processes. These latter regimes may be called pluralist authoritarianisms (Szmolka, 2010). Other authors have referred to them as "competitive authoritarianisms" (Levitsky and Way, 2002; Diamond, 2002). However, this term emphasizes a property of the political systems, political competition, which is limited in the authoritarianism regimes. So, the term "pluralist" may be more appropriate, since it refers to the possibility of political confrontation by various political opponents.

So, whereas in democracies, the only restriction established to pluralism and politi- cal participation is that implying a violation of legal and constitutional principles, in pluralist authoritarian regimes, some of the political forces may be relegated from the political processes, with political competition being thereby limited. Also, the proper functioning of the government may be altered by the lack of autonomy of the institutions, a lack of balance or counterbalances between the powers or the presence of influential actors without political responsibility or formal actors who centralize the institutional and political process, to the detriment of representative institutions. On the other hand, although the Rule of Law has been formally recognized, in pluralist authoritarianism regimes, a limitation of rights and freedoms often exists, placing the political power foundations at risk.

Given their level of political competition, the pluralist authoritarianism regimes may be considered quasi-competitive or hegemonic. The main difference between them lies in the greater irregularities of the elections, the ultra-dominant position of one specific party or coalition in the political processes and the practical impossibility of governmental alteration by the ballot box, produced in hegemonic pluralist authoritarianisms (Szmolka, 2010).

Despite the differences that may exist between the democracies and the pluralist authoritarianisms, in fact, the functions carried out by the parties in both regimes are quite similar: interest aggregation and social demands, representation, political personnel recruitment, mobilization, socialization, participation, government formation and creation of public policies (Hinnebush, 2017; Storm, 2017; Cavatorta and Storm, 2018).

This justifies the relevance and appropriateness of also examining the party systems of pluralist authoritarianism regimes. However, only Sartori (1976) created a classification of the party systems in non-democratic regimes, differentiating between single party 
and hegemonic party systems 5 . The single party system category is not relevant from a theoretical approach, given the existence of at least some pluralism and political competition. On the other hand, the hegemonic party system category of Sartori, in which one party has power and the other authorized political forces act as mere satellites with no real opportunity for change, does not exhaust the potential cases that may currently exist in which the competition for power may be greater and that may even permit a change in power under the limits imposed by the regime. Similarly, the party systems in pluralist authoritarianism regimes also present differences in terms of ideological divisions or other relevant social cleavages, or based on the established idea that this party system is a part of the political regime.

Therefore, below we present a general typology of party systems for application, regardless of the type of political regime considered and that includes the most relevant dimensions of the party systems.

\section{Party system typology PROPOSAL}

The proposed typology is based on the consideration of the multi-dimensional nature of the party system (Gross and Sigelman, 1984: 463; Wolinetz, 2004 and 2006; Bardi and Mair, 2008: 149; Croissant and Völkel, 2016: 237). For this, the typology includes four key dimensions of the party systems: competition, stability of the party system, number and balance between the parties and polarization (Table 1).

\section{Party competition}

Given that the purpose of this article is to offer a typology that is applicable to both democratic as well as authoritarian regimes,

\footnotetext{
5 Caramani (2011: 319) reproduces the classification created by Sartori for authoritarian regimes under the same terms.
}

the first definitive criterion to be considered is party competitiveness, understood to be the party's capacity to fight for and access power. The degree of competitiveness is determined by the following variables:

1) Political pluralism. The legal recognition of the political parties and/or movements is examined, as well as the formal or practical limits to party creation and action, the representativeness of the parties based on the relevant social cleavages (ideological, religious, ethnic, territorial, etc.) and the potential exclusion of parties from the political system. Political pluralism is analyzed through constitutions, laws on parties and party prohibitions or outlawing.

2) Electoral integrity. The objective is to determine whether or not the elections are free and clean and whether they permit representative parliaments and the formation of democratic governments. Electoral integrity is measured through the Perceptions of Electoral Integrity Index (PEI) created by Harvard University and the University of Sydney. This index offers complete coverage of all countries holding elections and includes all of the phases of the electoral cycle. The PEI considers electoral integrity as one of the following: very high, when over 70 points; high, 69-60; moderate, 59-50; low, 49-40; and very low, less than 40.

3) Governmental alternation. It is determined whether or not changes have taken place in the parties leading the government formation following the elections or, at least, if there is the possibility that this will occur.

Based on these criteria, the following party systems are considered:

a) Competitive. Permit free political play and the exercising of real governmental opposition. The integrity of the electoral processes is high or very high, therefore governmental alternation possibilities are real.

b) Quasi-competitive. There is legal recognition of the parties and a broad spec- 
trum of political forces having the ability to dispute power and be represented in the institutions participate in the political system. Opposition parties are present in the institutions, can criticize the government and propose alternative programs. However, certain parties may be excluded by the regime or may not be permitted to participate if it is considered that democratic conditions do not allow this. On the other hand, elections take place without serious accusations of fraud, although some irregularities are observed that favor the official parties (moderate electoral integrity). Alternation in the government is possible according to election results.

c) Hegemonic. The competition is limited by the hegemonic position of a certain party, group or coalition in the system and the political processes, by the obstacles to opposition activity and by serious irregularities in the electoral processes (low or very low electoral integrity). In this way, interaction of parties in the political system is permitted, but not real competition in terms of access to power, since there is no possibility of governmental alternation.

d) Non-pluralist. The competition is annulled by the absence of pluralism and the possibility of access to power by election. The existence of political groups or parties that are distinct from the ones in power are not permitted, therefore, it is impossible to discuss a party system.

\section{Stability of party system}

The second dimension assumes the dichotomy proposed by Sartori (1976: 310) between crystallized and fluid party systems, based on their development and continuity over time. Mainwaring and Scully (1995) applied the concept of institutionalization to party systems, distinguishing between insti- tutionalized and incipient party systems 6 . They noted four characteristics of institutionalized party systems: stability in the rules and in the nature of the partisan competition, stable roots in the society, legitimacy of the parties and of the elections and strength of the party organization.

Institutionalization of the party system is, therefore, a multi-dimensional concept that Mainwaring and Scully measure with indicators such as electoral volatility, difference in votes between the presidential and legislative elections, data from surveys and qualitative information on party adherence, seniority of the parties and qualitative evidence on the party organization. Based on Mainwaring and Scully's criteria, other forms of measuring the concept of more systematic institutionalization have been created, such as that by Jones (2007) and Croissant and Völkel (2012). Similarly, Lindberg (2007) used eight indicators to categorize the party systems as destabilized, fluid or stable.

The analysis of a multi-dimensional concept as complex as that of institutionalization exceeds the purposes of this article which are to construct a basic classification of party systems. In addition, many of the Mainwaring and Scully conditions are less determinant. So, an institutionalized party system with low confidence and party identification is compatible. On the other hand, the party organization refers to the party as a "unit" and not as a "system" (Randall and Svasand, 2002: 5).

In this way, the proposed typology focuses on the following elements that are related to party system continuity:

1) Origin of the party system. It dates back to the year when the first pluralist legis-

\footnotetext{
6 The relevance attributed to the institutionalization of the party system comes from its relationship with democratic consolidation. However, its analysis is also relevant in pluralist authoritarian regimes since an institutionalized party system may influence the resilience of the authoritarianism (Hicken and Martínez-Kuhonta, 2011: 573).
} 
lative elections of the current political regime were held. A threshold of 20 years is established, the period in which typically, at least four elections are held, so as to differentiate between the party systems with a non-recent or extended time trajectory.

2) Stability or change in party offer. On the one hand, the percentage of new parties appearing in the elections is used as an indicator (new formations, merged or divided parties), taking into account a percentage over $15 \%$ as a relevant change in the party offer. On the other hand, the Pedersen aggregate electoral volatility index ${ }^{7}$ was applied, assuming that, in a stable party system, the electoral volatility should be low (under $15 \%)^{8}$ or circumstantial (in a certain electoral process).

3) Stability or change of parliamentary parties. Similarly, the percentage of new parliamentary parties and the aggregate volatility are used in their parliamentary dimension.

Based on this, the party systems are categorized as follows:

a) Stable. The vast majority of the parties have an extensive and continuous trajectory in the political system, the partisan offer is continuous and the volatility is low or circumstantial.

b) Fluid. Although the party system may have a lengthy origin in terms of time, the reconfigurations of the party scene are constant and therefore, reorientations in the vote take place in each election and in the parliamentary party system.

c) Unstructured. The party system is recently developed. Of the few electoral processes held, there is a high level of change in the electoral offer and in the parties with parliamentary representation.

\footnotetext{
7 The Pedersen index (1979) is calculated from the sum of the absolute percentages of votes or seats of each party from one election to another, divided by two.

8 Ersson and Lane (1998).
}

\section{Number and balance between parties}

The numeric format of the party system influences aspects such as vote orientation, electoral participation, political representation, government formation and stability, political coalitions, the generation of consensus or political conflict (Dalton, 2008: 902; Ruiz and Otero, 2013: 125). Similarly, in the non-democratic context, partisan division may serve as an instrument for the control of power when used by authoritarian leaders.

There are distinct forms of quantifying the number of parties in the empirical analysis. In a pioneering study, Duverger (1954) distinguished between single, bipartisan, and multi-party systems in relation to the number of existing parliamentary parties. And Blondel (1968) examined the percentage of votes obtained by the two main parties and the distribution of seats. On this base, it was possible to identify bipartisan systems, of two and a half parties, multi-party systems with a predominant party and multi-party without a predominant party systems. Later, Ware (1996: 239) considered those parties that exceeded $3 \%$ of the seats, as a criterion for determining the number of parties, situating the analysis of the party systems within an institutional sphere. However, any exclusion threshold was arbitrary (Golosov, 2010: 172), and failed to reflect the influence of each party of the system (Siaroff, 2003: 268). So, according to Sartori (1976), a party "counted" if it affected the mechanism and functioning of the system due to its potential for coalition or blackmail potencial. However, this criterion is difficult to operationalize. Thus, distinct forms have been created to account for the party systems, considering the relative weight of each formation. The most frequently used index is the Effective Number of Parliamentary Parties (ENPP) or Electoral Parties (ENEP) by Laakso and Taagepera (1979)9.

\footnotetext{
9 The ENP is a measure that offers the relative weight of each electoral or parliamentary party based on their
} 
Finally, the classification created by Siaroff (2000: 69-72; 2003: 271) for party systems is quite useful, since it considers both the number of parties as well as the balance between them, permitting the differentiation of various categories of moderate or highly fractioned multi-party systems and the relevance of the majority parties. As indicators, Siaroff used the following: the number of parties with at least $3 \%$ of the seats (P3\%S); parliamentary concentration of the two majority parties (2PSC); the ratio of seats between the first and second parties (SR1:2); the ration between the second and third parties (SR2:3); and the mean of the ENPP in which the analyzed cases are situated. Based on this, Siaroff (2000) classified the party systems as follows:

a) Bipartisan. The two main parties have at least $95 \%$ of the seats, with a balance existing between them. Normally, one party creates an absolute majority. The number of parties with over $3 \%$ seats is two or three and the ENPP of the analyzed cases is situated at mean values of 1.92 .

b) Two and a half parties. The two main parties receive between $80-95 \%$ of the seats, the ration between the first and second party is less than 1.6 and the ration between the second and third is 1.8 or greater. It is likely that one of the two main parties will have the absolute majority and that between 3 and 5 (normally 3 or 4 ) parties will surpass $3 \%$ of the seats (ENPP mean of 2.56).

c) Moderate multi-party with one dominant party. In which three to five parties exceed $3 \%$ of the seats and the ration between the first and the second party is 1.6 or greater. It is possible that the main par-

relative strength (in votes or seats). The formula is $\mathrm{N}=1 / \sum_{i=1}^{n} p_{i}^{2}$, where $\mathrm{N}$ is the number of parties with votes or seats and $p_{i}^{2}$ is the squared proportion of votes/seats of each party. ty has absolute majority (ENPP mean of 2.95).

d) Moderate multi-party with two main parties. Between three and five parties exceed $3 \%$ of the seats, with the ration between the first and the second party being less than 1.6 and the ration between the second and third party being 1.8 or greater (ENPP mean of 3.17).

e) Moderate multi-party with balance between parties. Between three and five parties exceed $3 \%$ of the seats, with the ration between the first and the second party being less than 1.6 and the ration between the second and third party being less than 1.8 (ENPP mean of 3.69).

f) Extreme multi-party with one dominant party. Over five parties exceed $3 \%$ of the seats and the ration between the two main parties is greater than 1.6 (ENPP mean of 3.96).

g) Extreme multi-party with two main parties. Over five parties receive $3 \%$ of the seats, with the ration between the two main parties being less than 1.6 and the ration between the second and third party being 1.8 or greater. Although this is not a definitive trait, the parliamentary concentration of the two main parties is situated between 55 and $75 \%$. None of the two parties has absolute majority (ENPP mean of 4.41).

h) Extreme multi-party with balance between the parties. There are more than five parties with $3 \%$ of the votes, with the ration between the two main parties being less than 1.6 and the ration between the second and third party being less than 1.8. The concentration of the two main parties tends to be less than $60 \%$ (ENPP mean of 5.56).

In the proposed classification, the indicators and categories created by Siaroff are used as follows: first, the numeric format of the party system is determined by calculating the ENPP. Since the ENPP offered by Siaroff 
for each category of party systems includes a mean of the cases analyzed, the conversion by Mainwaring and Scully (1995: 31-32) is used, which grants the following: between 1.8-2.4, a bipartisan system; between 2.52.9 , a two and a half party system; between 3-3.9, a limited multi-partisan system; and over 4 points, an extreme multi-partisan system. In the case of the bipartisan and two and a half systems, the concentration of seats is also considered, which is the criteria that best identifies these categories, using the Siaroff thresholds. Second, the balance of the party systems is analyzed: determining whether there is a dominant party scenario (SR1:2 $\geq 1.6)$ or not $(<1.6)$ and, if not, verifying whether there is predominance of two parties (SR1:3 $\geq 1.8)$ or a balance between parties $(<1.8)$.

\section{Polarization}

The relevance of the analysis of the polarization of the party system lies in its influence on party competition, electoral behavior, government formation and stability or the adoption of agreements between parties, among other aspects (Sani and Sartori, 1983: 337; Ocaña and Oñate, 1999: 237). Similarly, this polarization may influence the success or failure of the democratization processes (Szmolka 2017: 349).

Sartori (1976) was the first to consider ideological polarization as a differentiating element of party systems. He tended to identify polarization with the extreme multi-party systems, due to dynamics of centrifugal competition and anti-systemic parties. Other authors have also considered polarization as being the ideological distance between parties (Sigelman and Yough, 1978; Sani and Sartori, 1983; Hazan, 1997; Dalton, 2008). However, ideology does not always describe the dimension by which parties (or voters) are divided, but rather, it may be necessary to consider other cleavages (ethnic, linguistic, religious, territorial, etc.), or a specific com- bination of these (Stoll, 2008: 1445; Lauka, McCoy and Firat, 2018: 3).

On the other hand, polarization also refers to the possible grouping of parties in blocks. So, Wolinetz $(2004,2006)$ when considering the party systems, takes into account the number of poles (unipolar, bipolar or multipolar) as well as the presence/ absence of permanent or semi-permanent alliances between the parties (clustering). Caramani (2011: 329 and 332) considers bipolar systems as a specific category of the party systems, determined by the existence of two large coalitions that total approximately $80 \%$ of the votes and seats; the balance in electoral and parliamentary weight (around $40-50 \%$ of the vote or of the seats); the stability of the coalitions and their concurrence in electoral alliances; the alternation in the government between coalitions; and the formation of coalition governments.

Diverse indicators have been used to measure polarization, such as the location of the parties on an ideological, left-right spectrum or in political families (Sartori, 1976; Sigelman and Yough, 1978; Gross and Sigelman, 1984) or partisan positions through their political programs (Budge, Robertson and Hearl, 1987; Wildford, 2017). These forms of measurement have the inconvenience of not considering the relative weight of each party (Ocaña and Oñate, 1999: 237). Therefore, indexes have been created to calculate polarization, such as those by Taylor and Herman (1971), Knutsen (1998) or Dalton (2008: 904).

In the case of the proposed classification, polarization is measured based on the following variables:

1) Distance between parliamentary parties. If there is data on party positioning, the Dalton polarization index is used. In addition, or as a substitute, the identification of the parties in political families is considered, paying special attention to the presence of 
parties on the ideological extremes (having a parliamentary representation of greater than 10\%) and/or anti-systemic parties. However, the latter indicator is not determinant, given that there may be a polarization scenario with an absence or presence of anti-systemic parties. So, in an authoritarian context, their participation is practically impossible.

2) Stable or semi-stable party alliances with regards to concurrence to elections (with parliamentary representation), government formation (coalition governments or parliamentary support to their investiture) or relevant political processes (for example, political reforms).

3) Number of poles. In the case in which there is a scenario of partisan polarization and a collaboration trajectory between parties, the number of poles in which the parties are segmented is determined.

So, the following are distinguished:

a) Unipolar non-polarized party systems. The large majority of parties, including the most important parties, are situated in the center or in positions close to the center.

b) Bipolar polarized party systems. The parties are grouped in two balanced blocks (similar percentage of seats) and are opposing with regards to ideology or any other cleavage. The creation of permanent or semi-permanent party coalitions or alliances is typical (electoral, parliamentary or governmental).

c) Multipolar polarized party systems. The parties are grouped together in central and extreme positions (on both sides). The establishment of partisan coalitions or alliances is typical (electoral, parliamentary or governmental) which may be changing.

\section{Classification and CHARACTERIZATION OF PARTY SYSTEMS OF MOROCCO, Algeria AND TUnisia}

\section{Partisan competition}

The perception of extensive pluralism in Maghreb is a result of data on the number of parties that participated in the last legislative elections of these countries: 27 parties in the 2016 elections in Morocco; 69 in Algeria in 2017 (more than 163 independent lists); and, up to 890 in Tunisia in 2014 (more than 151 coalitions and 459 independent lists).

TABLE 1. General and multi-dimensional classification of party systems

\begin{tabular}{|c|c|c|c|c|}
\hline $\begin{array}{l}\text { Dimen- } \\
\text { sions }\end{array}$ & Variables & Indicator & Measurement & Classification by categories \\
\hline \multirow{5}{*}{ 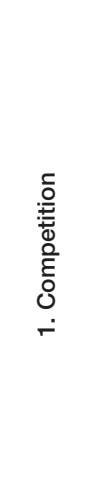 } & \multirow{3}{*}{$\begin{array}{l}\text { Political } \\
\text { pluralism }\end{array}$} & Legal recognition of parties & & \\
\hline & & $\begin{array}{l}\text { Legal restrictions to party } \\
\text { creation }\end{array}$ & $\begin{array}{l}\text { No pluralism/ Limited } \\
\text { pluralism/ Unlimited }\end{array}$ & $\begin{array}{l}\text { Competitive: unlimited pluralism, high } \\
\text { or very high electoral integrity, real or } \\
\text { factual governmental alternation }\end{array}$ \\
\hline & & $\begin{array}{l}\text { Parties excluded or } \\
\text { self-excluded from the } \\
\text { political system }\end{array}$ & pluralism & $\begin{array}{l}\text { Quasi-competitive: limited pluralism, } \\
\text { moderate electoral integrity, real or } \\
\text { factual governmental alternation }\end{array}$ \\
\hline & $\begin{array}{l}\text { Electoral } \\
\text { integrity }\end{array}$ & $\begin{array}{l}\text { Perceptions of Electoral } \\
\text { Integrity Index (PEI) }\end{array}$ & $\begin{array}{c}\text { Very high }(\geq 70) / \text { High } \\
(69-60) / \text { Moderate }(59-50) / \\
\text { Low }(49-40) \text { / Very low }(<40)\end{array}$ & $\begin{array}{l}\text { Hegemonic: limited pluralism, low or } \\
\text { very low electoral integrity, inexistence } \\
\text { of governmental alternation }\end{array}$ \\
\hline & $\begin{array}{l}\text { Change in } \\
\text { government }\end{array}$ & $\begin{array}{l}\text { Change in parties in the } \\
\text { governmental formation }\end{array}$ & Yes/No & Non-competitive: non-pluralist \\
\hline
\end{tabular}


TABLE 1. General and multi-dimensional classification of party systems. (Continuation)

\begin{tabular}{|c|c|c|c|c|}
\hline $\begin{array}{l}\text { Dimen- } \\
\text { sions }\end{array}$ & Variables & Indicator & Measurement & Classification by categories \\
\hline \multirow{6}{*}{ 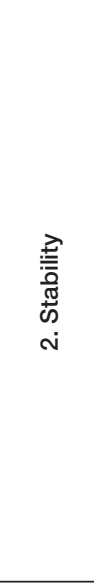 } & $\begin{array}{l}\text { Origin of the } \\
\text { party } \\
\text { system }\end{array}$ & $\begin{array}{l}\text { Year of the first legislative } \\
\text { elections (current regime) }\end{array}$ & $\begin{array}{l}\text { Recent }(<20 \text { years }) / \\
\text { Extended }(>20)\end{array}$ & \multirow{4}{*}{$\begin{array}{l}\text { Stable: extended origin, crystallized } \\
\text { electoral offer, continuity in parliamen- } \\
\text { tary composition and low or } \\
\text { circumstantial volatility } \\
\text { Fluid: extended origin, changing } \\
\text { partisan offer and parliamentary } \\
\text { composition and high volatility }\end{array}$} \\
\hline & \multirow{3}{*}{$\begin{array}{l}\text { Stability or } \\
\text { change in } \\
\text { electoral } \\
\text { offer }\end{array}$} & $\begin{array}{l}\text { Percentage of new parties } \\
\text { over the total presenting in } \\
\text { the elections }\end{array}$ & $\begin{array}{l}\text { Crystalized electoral party } \\
\text { offer }(<15 \%) / \text { fluid ( } \geq 15 \%)\end{array}$ & \\
\hline & & \multirow{2}{*}{$\begin{array}{l}\text { Pedersen's index of } \\
\text { electoral volatility }\end{array}$} & High volatility (>15) / & \\
\hline & & & $\begin{array}{c}\text { Moderate or low }(<15) / \\
\text { Circumstantial (volatility }>15 \\
\text { in a single election) }\end{array}$ & \\
\hline & \multirow{2}{*}{$\begin{array}{l}\text { Stability or } \\
\text { change in } \\
\text { the } \\
\text { parliamen- } \\
\text { tary parties }\end{array}$} & $\begin{array}{l}\text { Percentage of new } \\
\text { parliamentary parties }\end{array}$ & $\begin{array}{l}\text { Parliamentary change } \\
(>15 \%) / \text { Stability }(<15 \%)\end{array}$ & \multirow{2}{*}{$\begin{array}{l}\text { Unstable: recent origin, changing } \\
\text { partisan offer and parliamentary } \\
\text { composition and high volatility }\end{array}$} \\
\hline & & $\begin{array}{l}\text { Pedersen's index of } \\
\text { parliamentary volatility }\end{array}$ & $\begin{array}{c}\text { High volatility }(>15) / \\
\text { Moderate or low }(<15) / \\
\text { Circumstantial (volatility }>15 \\
\text { in a single legislature) }\end{array}$ & \\
\hline \multirow{4}{*}{ 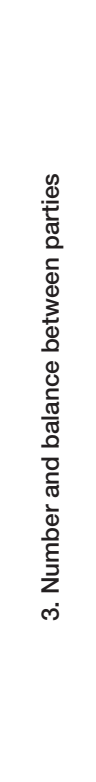 } & $\begin{array}{l}\text { Number of } \\
\text { parties }\end{array}$ & $\begin{array}{l}\text { Effective number of } \\
\text { parliamentary parties }\end{array}$ & $\begin{array}{c}\text { Bi-partisan (1.8-2.4); two } \\
\text { and a half (2.5-2.9); } \\
\text { moderate multi-party system } \\
\text { (between 3-3.9); extreme } \\
\text { multi-party system (>4) }\end{array}$ & \multirow{4}{*}{ 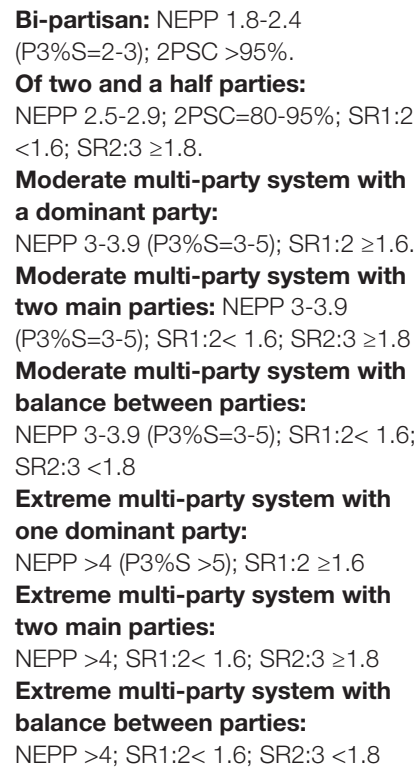 } \\
\hline & \multirow{3}{*}{$\begin{array}{l}\text { Predomi- } \\
\text { nance/ } \\
\text { Balance } \\
\text { between } \\
\text { parties }\end{array}$} & Parliamentary concentration & 2PSC (>95\%; 80-95\%) & \\
\hline & & Dominance of one party & SR $1: 2<1.6$ and SR $2: 3 \geq 1.8$ & \\
\hline & & Balance between parties & $\mathrm{SR} 1: 2<1.6$ and SR2:3 $<1.8$ & \\
\hline \multirow{5}{*}{ 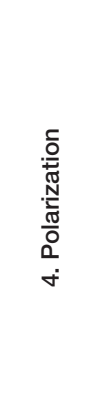 } & \multirow{3}{*}{$\begin{array}{l}\text { Distance } \\
\text { between } \\
\text { parties }\end{array}$} & Dalton's polarization index & & \multirow{5}{*}{$\begin{array}{l}\text { Not polarized unipolar } \\
\text { Polarized bipolar } \\
\text { Polarized multipolar }\end{array}$} \\
\hline & & $\begin{array}{l}\text { Parliamentary parties of } \\
\text { ideological extremes } \\
\text { (with over } 10 \% \text { seats) }\end{array}$ & Polarized/ Not polarized & \\
\hline & & $\begin{array}{l}\text { Existence or not of } \\
\text { anti-systemic parties }\end{array}$ & & \\
\hline & $\begin{array}{l}\text { Party } \\
\text { coalitions }\end{array}$ & $\begin{array}{l}\text { Formation or not of electoral, } \\
\text { governmental or political co- } \\
\text { alitions (with representation), }\end{array}$ & $\begin{array}{l}\text { Non-existent/ Stable/ } \\
\text { Circumstantial }\end{array}$ & \\
\hline & Poles & $\begin{array}{l}\text { Number of polies in which } \\
\text { the parties are grouped }\end{array}$ & Unipolar/ Bipolar/ Multipolar & \\
\hline
\end{tabular}

Source: Author's own creation. 
However, in these three cases, the pluralism is not absolute. The democratic transition in Tunisia, following the fall of the Ben Ali regime in 2011, permitted the freedom of party creation with the sole exclusion of those parties that infringe upon the identity or integrity of the state. On the other hand, despite a wide variety of political forces that span the ideological, left-right spectrum, in Morocco and Algeria, the creation of parties that are based on religion, language, race or region are prohibited. So, various parties or movements have not been authorized or have dissolved (see Table 2). Despite this, it is possible to identify parties having Berber leaders and social support: in Morocco, the Popular Movement; and in Algeria, the Socialist Forces Front and the Rally for Culture and Democracy. Similarly, there are some Islamist parties that were legalized and that currently have parliamentary representation: the Justice and Development Party (JDP) in Morocco; the Movement of Society for Peace
(MSP), Islah and Ennahda in Algeria; and Ennahda and Hizb ut-Tahir in Tunisia.

On the other hand, pluralism is not synonymous with competition. In addition to restrictions on party creation, the electoral processes of Morocco and Algeria have suffered from irregularities. According to the PEI, during 2012-2018, the elections held in Tunisia had a high level of integrity, while those in Morocco had a moderate integrity level and those in Algeria had a low integrity level.

Finally, governmental alternation has been possible in Morocco and in Tunisia on two occasions, but has never occurred in Algeria. In Morocco, in 1998, the opposing party, the Socialist Union of Popular Forces (SUPF) formed government with another six political forces; and in 2012, the Islamic party JDP, ruled with a governmental coalition with three other parties. However, on both occasions, there was "cohabitation" of opposition parties with the ruling parties. In Tunisia, governmental alternation took place as

TABLE 2. Party competition

\begin{tabular}{|c|c|c|c|c|}
\hline Variable & Indicator & Morocco & Algeria & Tunisia \\
\hline \multirow{3}{*}{$\begin{array}{l}\text { Political } \\
\text { pluralism }\end{array}$} & $\begin{array}{l}\text { Legal } \\
\text { recognition of } \\
\text { parties }\end{array}$ & $\begin{array}{l}\text { Organic Law 29-11, } 23 \\
\text { October } 2011\end{array}$ & $\begin{array}{l}\text { Organic Law 4-12, } 15 \\
\text { January } 2012\end{array}$ & $\begin{array}{l}\text { Decree-Law 2011-87, } \\
24 \text { September } 2011\end{array}$ \\
\hline & $\begin{array}{l}\text { Legal } \\
\text { restrictions }\end{array}$ & $\begin{array}{l}\text { Religious, linguistic, ethnic } \\
\text { or regional parties }\end{array}$ & $\begin{array}{l}\text { Religious, linguistic, } \\
\text { racial, sexual, corporate } \\
\text { or regional parties }\end{array}$ & No \\
\hline & $\begin{array}{l}\text { Prohibited } \\
\text { parties/ } \\
\text { movements, } \\
\text { unauthorized or } \\
\text { self-excluded }\end{array}$ & $\begin{array}{l}\text { Berbers: Moroccan } \\
\text { Amazigh Democrat Party } \\
\text { Islamic: al-Umma; Al-Badil } \\
\text { al-Hadari; Al-Adl wa al-Ihsan } \\
\text { party } \\
\text { Extreme leftist: Annahj } \\
\text { Addimocrati }\end{array}$ & $\begin{array}{l}\text { Islamist: Islamic Salvation } \\
\text { Front; Movement for } \\
\text { Democracy; Wafa'; Hizb } \\
\text { ut-Tahir } \\
\text { Leftist: Arab Socialist } \\
\text { Ba'ath Party }\end{array}$ & - \\
\hline $\begin{array}{l}\text { Electoral } \\
\text { integrity }\end{array}$ & PEI & Moderate (56) & Low (43) & High (67) \\
\hline $\begin{array}{l}\text { Governmental } \\
\text { change }\end{array}$ & $\begin{array}{l}\text { Change in } \\
\text { parties in the } \\
\text { government } \\
\text { formation }\end{array}$ & $\begin{array}{l}\text { 1998: Yussufi government } \\
\text { (USFP in coalition) } \\
\text { 2011: Benkiran government } \\
\text { (PJD in coalition) }\end{array}$ & No & $\begin{array}{l}\text { 2011: Ghannuchi } \\
\text { government (Ennahda in } \\
\text { coalition) } \\
\text { 2014: Chahed government } \\
\text { (Nidaa Tounes in coalition) }\end{array}$ \\
\hline Classification & & Quasi-competitive & Hegemonic & Competitive \\
\hline
\end{tabular}

Source: Author's own creation. 
a result of the democratic transition. Following the elections to the Constituent Assembly of 2011, the winning party, Ennahda, formed government together with three other parties. The second governmental alternation took place in 2014 when the national unity movement Nidaa Tounes led a coalition government with Ennahda and two minority parties. On the other hand, in Algeria, no governmental changes have taken place since the civil war, with the government remaining in the hands of the coalition formed by the $\mathrm{Na}$ tional Liberation Front (FLN), the Democratic National Rally (RND) and, until just before the 2017 elections, the MSP.

Ultimately, the analysis carried out characterizes the party system as competitive in
Tunisia, quasi-competitive in Morocco and hegemonic in Algeria.

\section{Stability of the political systems}

The origin of the multi-party system is distinct in the three cases under study. Unlike the single party systems of the post-colonial regimes of Algeria (FLN) and Tunisia (Neodestur party), in Morocco, the multi-party system was introduced following its independence in 1956, with the first legislative elections taking place in 1962. The participation of other parties in the Tunisian and Algerian political systems did not take place until 1983 and 1989, respectively. However, the civil war occurring in Algeria in 1991 led to an institutional gap

TABLE 3. Stability of the party system

\begin{tabular}{|c|c|c|c|c|}
\hline Variable & Indicator & Morocco & Algeria & Tunisia \\
\hline Origin of party system & $\begin{array}{c}\text { Year of first legislative } \\
\text { elections }\end{array}$ & $\begin{array}{c}1962 \\
\text { Dilated }\end{array}$ & $\begin{array}{c}1997 \\
\text { Dilated }\end{array}$ & $\begin{array}{c}2011 \\
\text { Recent }\end{array}$ \\
\hline \multirow{10}{*}{$\begin{array}{l}\text { Stability or change in } \\
\text { electoral offer }\end{array}$} & \multirow{5}{*}{$\begin{array}{c}\text { New electoral parties } \\
(\%)\end{array}$} & 16.6 (2016-2011) & 37.7 (2017-2012) & \multirow[t]{4}{*}{$94.4(2014-2011)$} \\
\hline & & 18.9 (2011-2007) & 47.7 (2012-2007) & \\
\hline & & 22.0 (2007-2002) & $4.2(2007-2002)$ & \\
\hline & & & $17.4(2002-1997)$ & \\
\hline & & Fluid offer & Fluid offer & Fluid offer \\
\hline & \multirow[t]{5}{*}{ Electoral volatility } & 15.7 (2016-2011) & 53.9 (2017-2012) & \multirow[t]{4}{*}{76.4 (2014-2011) } \\
\hline & & 26.8 (2011-2007) & 43.3 (2012-2007) & \\
\hline & & 17.7 (2007-2002) & $41.0(2007-2002)$ & \\
\hline & & & 58.6 (2002-1997) & \\
\hline & & High & High & High \\
\hline \multirow{10}{*}{$\begin{array}{l}\text { Stability or change in } \\
\text { parliamentary parties }\end{array}$} & \multirow{5}{*}{$\begin{array}{l}\text { New parliamentary } \\
\text { parties (\%) }\end{array}$} & $0(2016-2011)$ & 34.4 (2017-2012) & \multirow[t]{4}{*}{$60.0(2014-2011)$} \\
\hline & & 33.3 (2011-2007) & 38.5 (2012-2007) & \\
\hline & & 26.1 (2007-2002) & 68.2 (2007-2002) & \\
\hline & & & 44.4 (2002-1997) & \\
\hline & & From change to stability & Change & Change \\
\hline & \multirow[t]{5}{*}{ Parliamentary volatility } & $19.2(2016-2011)$ & 38.6 (2017-2012) & \multirow[t]{4}{*}{$60.5(2014-2011)$} \\
\hline & & 26.0 (2011-2007) & 33.7 (2012-2007) & \\
\hline & & 21.6 (2007-2002) & 39.9 (2007-2002) & \\
\hline & & & $58.6(2002-1997)$ & \\
\hline & & High & High & High \\
\hline Clasification & & Fluid & Fluid & Unstructured \\
\hline
\end{tabular}

Source: Author's own creation. 
until the celebration of the pluralist presidential elections in 1995 and the legislative elections of 1997. And, in Tunisia, the democratic transition process as a result of the Arab Spring, allowed for the holding of the first competitive elections in October of 2011. In summary, the current party systems have a recent parliamentary trajectory of 7 years in Tunisia and an extended one of 56 years in Morocco and 21 years in Algeria.

On the other hand, the electoral offer has been quite changing in Algeria and in Tunisia, as revealed by the variation in the number of parties presenting themselves to the elections and the high electoral volatility. In Tunisia, this has been a consequence of the democratic transition, which allowed for the creation of new parties, not only in the face of the elections held that year, but also in those of 2014. Similarly, the Arab Spring led to an increase in new parties in Algeria, although the high electoral volatility is a characteristic element of the Algerian party system throughout the period. Despite this, these changes have not affected the hegemony of the two majority parties (FLN and RND) in Algeria. As for Morocco, despite its more extensive partisan trajectory, the high electoral volatility and the changing correlation of parliamentary forces continue to be significant characteristics. However, in 2016 no new parties entered parliament, although six parties from the prior legislature disappeared (Table 3).

Given all of this, the party systems of Morocco and Algeria have been characterized as fluid, while the Tunisia system has been characterized as unstructured.

\section{Number and balance between parties}

The three countries under study share an electoral system that is proportional to the majority (Hare quota) having multi-member constituencies in which closed and blocked lists exist. However, the parliamentary fragmentation is greater in Morocco and Algeria as compared to Tunisia.

So, the current party systems of Morocco and Algeria may be categorized as extreme multi-party systems with two main parties. In both cases, the parliamentary fragmentation is high, with the ENPP having a value of almost five parties. However, in Morocco, the fragmentation has decreased considerably since reaching its highest level in the 20072011 legislature (ENPP 10.4 parties). On the other hand, the absence of absolute majorities, the high concentration of seats amongst the two main parliamentary parties and the high ratio between the second and third party in both countries reveals the supremacy of the two majority parties (see Table 4). How-

TABLE 4. Number and balance between parties

\begin{tabular}{|c|c|c|c|c|c|c|c|c|c|c|c|}
\hline & \multicolumn{4}{|c|}{ Morocco } & \multicolumn{5}{|c|}{ Algeria } & \multicolumn{2}{|c|}{ Tunisia } \\
\hline & 2016 & 2011 & 2007 & 2002 & 2017 & 2012 & 2007 & 2002 & 1997 & 2014 & 2011 \\
\hline P3\%S & $8 / 12$ & $8 / 18$ & $10 / 23$ & $11 / 22$ & $7 / 34$ & $5 / 27$ & $7 / 23$ & $5 / 10$ & $6 / 10$ & $5 / 15$ & $5 / 19$ \\
\hline NEPP* $^{*}$ & 5.0 & 6.6 & 10.4 & 10.1 & 4.8 & $\begin{array}{l}3.8 \\
4.1\end{array}$ & $\begin{array}{l}4.8 \\
5.5\end{array}$ & 2.8 & 3.9 & 3.6 & 4.3 \\
\hline 2PSC & 57.5 & 42.3 & 40.0 & 30.1 & 56.9 & 59.7 & 50.6 & 63.2 & 58.9 & 71.4 & 54.4 \\
\hline SR1:2 & 1.2 & 1.8 & 1.1 & 1.0 & 1.6 & 3.1 & 2.2 & 4.2 & 2.2 & 1.2 & 3.1 \\
\hline SR2:3 & 2.2 & 1.1 & 1.1 & 1.1 & 3.0 & 1.4 & 1.2 & 1.1 & 1.1 & 4.3 & 1.1 \\
\hline Clasification & $\begin{array}{r}\text { Extrem } \\
\text { pred }\end{array}$ & $\begin{array}{l}\text { multi- } p \\
\text { inance }\end{array}$ & $\begin{array}{l}\text { y syster } \\
\text { two pa }\end{array}$ & & $\begin{array}{r}\text { Extreme } \\
\text { predom }\end{array}$ & $\begin{array}{l}\text { ti-party } \\
\text { nce of }\end{array}$ & $\begin{array}{l}\text { stem n } \\
\text { o partie }\end{array}$ & & $\begin{array}{l}\text { ate mu } \\
\text { lominar }\end{array}$ & $\begin{array}{l}\text { arty sy } \\
\text { of two }\end{array}$ & $\begin{array}{l}m \text { with } \\
\text { rties }\end{array}$ \\
\hline
\end{tabular}

* Independent candidates have not been considered in the calculation of the NEPP. In Algeria, they represent $6.1 \%$ in 2017 ; $8.5 \%$ in 2007; $7.7 \%$ in 2002; and, $2.9 \%$ in 1997. And, in Tunisia: $1.4 \%$ in the 2014 elections; and, $3.7 \%$ in 2011.

Source: Author's own creation. 
ever, while in Morocco the second party, the Authenticity and Modernity Party (PAM), is the main opposition party, in Algeria, the RND has formed a part of the coalition government since 1997 (Szmolka, 2018).

Tunisia, on the other hand, currently has a moderate multi-party system in which two parties predominate. Despite this, up to 15 parties are represented in its Parliament, although only 5 surpass $3 \%$ of the seats. Nidaa Tounes and Ennahda hold $71.4 \%$ of the seats and are quite distanced from the third-ranked party. Despite their ideological differences, both parties participate in the government coalition.

\section{Polarization}

Given the absence of data on the ideological positioning of the parties, it has been impossible to calculate the Dalton polarization index. So, alternative indicators have been used, such as the existence of ideologically extreme and anti-systemic parliamentary parties. As observed in Table 5, there are no extremist or anti-systemic parties with parliamentary representation in the three countries. In Morocco and Algeria, this is a result of the authoritarian characteristics of the respective political regimes, which forces the parties to moderate their political discourse and programs, even those parties that may be more critical such as the Islamist and leftist ones. In Tunisia, the existence of an Islamist party such as Ennahda polarizes the religious party scene, given its moderation since its origins and its governmental participation since 2011.

On the other hand, the formation of coalitions does not determine the existence of polarization. With regards to the electoral coalitions, two electoral coalitions received representation in Algeria in 2017, both of which were Islamist. However, the absolute majori-

TABLE 5. Polarization

\begin{tabular}{|c|c|c|c|c|}
\hline Variable & Indicator & Morocco & Algeria & Tunisia \\
\hline \multirow{2}{*}{$\begin{array}{l}\text { Distance } \\
\text { between parties }\end{array}$} & $\begin{array}{l}\text { Extreme parliamentary } \\
\text { parties }\end{array}$ & No & No & No \\
\hline & Anti-system parties & No & No & No \\
\hline \multirow[b]{2}{*}{ Coaliitions } & $\begin{array}{l}\text { Electoral (obtaining } \\
\text { representation) }\end{array}$ & No & $\begin{array}{l}\text { 2017: MSP-FC and } \\
\text { Ennahda-FJD } \\
\text { 2012: AW (MSP, } \\
\text { Ennahda, Islah) }\end{array}$ & Popular Front \\
\hline & Governmental & $\begin{array}{l}2018 \text { and } 2017 \text { : } \\
\text { PJD, RNI, MP, USFP, } \\
\text { PPS, UC } \\
\text { 2013: } \\
\text { PJD, RNI, MP, PPS } \\
\text { 2012: } \\
\text { PJD, PI, MP, PPS } \\
\text { 2007: } \\
\text { PI, RNI, USFP, PPS } \\
\text { 2002 y 2004: } \\
\text { USFP, RNI, MP, } \\
\text { MNP, UC }\end{array}$ & $\begin{array}{l}\text { 2017: } \\
\text { FLN, RND, TAJ, MPA } \\
\text { 2017-2012: } \\
\text { FLN, RND } \\
\text { 1997-2012: } \\
\text { FLN, RND, MSP }\end{array}$ & $\begin{array}{l}\text { 2016: Nidaa Tounes, } \\
\text { Ennahda, Afek Tounes, } \\
\text { UPL (merged with NT), } \\
\text { Movement of Socialist } \\
\text { Democrats, Al-Yumhuri } \\
\text { and al-Massar (out in } \\
\text { 2018) } \\
\text { 2015: Nidaa Tounes, } \\
\text { Ennahda, Afek Tounes, } \\
\text { UPL } \\
\text { 2011: Ennahda, CPR, } \\
\text { Ettakatol and PR }\end{array}$ \\
\hline Poles & & One & One & One \\
\hline Clasification & & Unipolar not polarized & Unipolar not polarized & Unipolar not polarized \\
\hline
\end{tabular}

Source: Author's own creation. 
ty of the FLN and the RND prevent bipolarization in Algeria. In Tunisia, the only noteworthy electoral coalition is that of the Popular Front, formed by nine parties, but only representing $5 \%$ of the seats. As for the governmental coalitions, in three countries, these are not alternating coalitions but rather, they are characterized by their over-dimensioned nature, with more parties than necessary participating, especially in Tunisia. Likewise, they are heterogeneous ideological and political coalitions in which Islamist parties coexist with the ruling parties and in Morocco and Tunisia, also with the moderate left.

In conclusion, the three party systems are characterized by being non-polarized, unipolar systems.

\section{Conclusions}

This article aims to provide a new typology of party systems in response to the scarce and limited classifications existing for this field, since the classic work of Sartori. The main contribution of the proposed typology is its potential application to both democratic and pluralist authoritarian regimes. In addition, it considers the main dimensions of the party systems: partisan competition, party system stability, number and balance between the parties and polarization. Thus, the typology permits comparative transnational research on party systems as well as a complete characterization of the same.
To verify its validity, the typology has been applied to three countries of Maghreb, allowing us to analyze party systems in three distinct political regimes having no prior classifications. The analysis of our study cases has led to the following characterization of party systems: Morocco, quasi-competitive party system, fluid, of extreme multi-party system with predominance of two parties and not polarized; Algeria, hegemonic party system, fluid, of extreme multi-party system with predominance of two parties and not polarized; and, Tunisia, competitive party system, non-structured of moderate multi-party system with predominance of two parties and not polarized.

The application of the typology is hindered by the lack of official data on the official registry of parties as well as incomplete electoral data; therefore, complementary sources were used such as ElectionGuide. Similarly, calculation of the Dalton polarization index was not possible since no data on party position were available. As an alternative, the classification of the parties in ideological families was used to determine the existence of political forces in the ideological extremes. On the other hand, working with periods presents certain difficulties, since the indices may vary in each of these, hindering the characterization of each dimension. However, variations were only found in one of the indicators of the stability of the party system dimension applied to Morocco, reflecting the tendency for change to stability in the parliamentary parties.

TABLE 6. Multi-dimensional classification of the party systems

\begin{tabular}{|c|c|c|c|c|}
\hline \multicolumn{5}{|c|}{ Party systems } \\
\hline & Competition & Stability & $\begin{array}{c}\text { Number and balance } \\
\text { between parties }\end{array}$ & Polarization \\
\hline Morocco & Quasi-competitive & Fluid & $\begin{array}{l}\text { Extreme multi-party system } \\
\text { with two main parties }\end{array}$ & Unipolar not polarized \\
\hline Algeria & Hegemonic & Fluid & $\begin{array}{c}\text { Extreme multi-party system } \\
\text { with two main parties }\end{array}$ & Unipolar not polarized \\
\hline Tunisia & Competitive & Not structured & $\begin{array}{c}\text { Moderate multi-party system } \\
\text { with two main parties }\end{array}$ & Unipolar not polarized \\
\hline
\end{tabular}

Source: Author's own creation. 
Therefore, the typology has revealed its utility as an instrument for the classification of party systems, serving as a valid starting point for comparative research. However, it would be useful to test this typology in other geographic areas, in order to verify its validity in other types of political regimes.

\section{BiBLIOGRAPHY}

Alcántara, Manuel; Campo, Esther del and Ramos, María Luisa (2001). "La naturaleza de los sistemas de partidos políticos y su configuración en el marco de los sistemas democráticos en América Latina". Justicia Electoral, 15: 57-83.

Bardi, Luciano and Mair, Peter (2008). "The Parameters of Party Systems”. Party Politics, 14(2): 147162.

Blondel, Jean (1968). "Party Systems and Patterns of Government in Western Democracies". Canadian Journal of Political Science, 1(2): 180-203.

Bogaards, Matthijs (2004). "Counting Parties and Identifying Dominant Party Systems in Africa". European Journal of Political Research, 43(2): 173-197.

Budge, lan; Robertson, David and Hearl, Derek (eds.) (1987). Ideology, Strategy and Party Change: Spatial Analysis of Postwar Election Programs in 19 Democracies. Cambridge: Cambridge University Press.

Caramani, Daniel (2011). Comparative Politics. Oxford: Oxford University Press.

Cavatorta, Francesco and Storm, Lise (2018). Political Parties in the Arab World. Continuity and Change. Edinburgh: Edinburgh University Press.

Coppedge, Michael (1998). "The Dynamic Diversity of Latin American Party Systems". Party Politics, 4(4): 547-568.

Croissant, Aurel and Völkel, Philip (2012). "Party Systems Types and Party System Institutionalization: Comparing New Democracies in East and Southest Asia". Party Politics, 18(2): 235-265.

Daalder, Hans and Mair, Peter (1983). Western European Party Systems. Beverly Hills, California/ London: Sage Publications.

Dalton, Russell (2008). “The Quantity and the Quality of Party Systems. Party System Polarization, Its Measurement, and Its Consequences". Comparative Political Studies, 41(7): 899-920.
Diamond, Larry (2002). "Elections Without Democracy. Thinking About Hybrid Regimes". Journal of Democracy, 13(2): 21-35.

Duverger, Maurice (1954). Political Parties: Their Organization and Activity in the Modern State. London: Methuen.

Erdmann, Gero and Basedau, Matthias (2008). "Political Parties in Africa: Problems of Categorising and Explaining Party Systems". Journal of Contemporary African Studies, 26(3): 241-258.

Ersson, Svante and Lane, Jan-Erik (1998). "Electoral Instability and Party System Change in Western Europe". In: Pennings, P. and Lane, J.-E. (eds.). Comparing Party System Change. London: Routledge.

Golosov, Gregorii (2010). "The Effective Number of Parties. A New Approach". Party Politics, 16(2): 171-192.

Golosov, Gregorii (2011). "Party System Classification. A Methodological Inquiry". Party Politics, 17(5): 539-560.

Gross, Donald and Sigelman, Lee (1984). "Party Systems: A Multidimensional Approach". Comparative Politics, 16(4): 463-479.

Hazan, Reuven (1997). Centre Parties. Polarization and Competition in European Parliamentary Democracies. London: Pinter.

Hicken, Allen and Martínez-Kuhonta, Eric (2011). "Shadows from the Past: Party System Institutionalization in Asia". Comparative Political Studies, 44(5): 572-597.

Hinnebush, Raymond (2017). "Political Parties in MENA: Their Functions and Development". British Journal of Middle Eastern Studies, 44(2): 159175.

Jones, Mark P. (2007). "Political Parties and Party Systems in Latin America". Paper presented at the Symposium Prospects for Democracy in Latin America. University of North Texas, Denton, Texas.

Knutsen, Oddbjorn (1998). "Expert Judgments of the Left-Right Location of Political Parties: A Comparative Longitudinal Study". West European Politics, 21(2): 63-94.

La Palombara, Joseph and Weiner, Myron (1966). Political Parties and Political Development. Princeton: Princeton University Press.

Laakso, Marku and Taagepera, Reinn (1979). “'Effective' Number of Parties: A Measure with Applica- 
tion to West Europe". Comparative Political Studies, 12(1): 3-27.

Lauka, Alban; McCoy, Jennifer and Firat, Rengin (2018). "Mass Partisan Polarization: Measuring a Relational Concept". American Behavioral Scientist, 62(1): 107-126.

Levitsky, Steven and Way, Lucan A. (2002). "Elections Without Democracy. The Rise of Competitive Authoritarianism". Journal of Democracy, 13(2): 51-65.

Lijphart, Arend (1994). Electoral Systems and Party Systems: A Study of Twenty-Seven Democracies, 1945-1990. Toronto: Oxford University Press.

Lindberg, Staffam (2007). "Institutionalization of Party Systems? Stability and Fluidity among Legislative Parties in Africa's Democracies". Government and Opposition, 42(2): 215-241.

Mainwaring, Scott (2018). Party Systems in Latin America. Cambridge: Cambridge University Press.

Mainwaring, Scott y Scully, Timothy (1995). "Party Systems in Latin America". In: Mainwaring, S. and Scully, T. (eds.). Building Democratic Institutions: Party-systems in Latin America. Stanford, California: Stanford University Press.

Mair, Peter (2002). "Comparing Party Systems". In: LeDuc, L., Niemi, R. and Norris, P. (eds.). Comparing Democracies 2: New Challenges in the Study of Elections and Voting. Thousand Oaks, California: Sage.

Ocaña, Francisco and Oñate, Pablo (1999). Análisis de datos electorales. Madrid: CIS.

Pedersen, Mogens (1979). "The Dynamics of European Party Systems: Changing Patterns of Electoral Volatility". European Journal of Political Research, 7(1): 1-26.

Pelizzo, Riccardo and Nwokora, Zim (2016). "Bridging the Divide: Measuring Party System Change and Classifying Party Systems". Politics and Policy, 44(6): 1017-1052.

Randall, Vickey and Svasand, Lars (2002). "Party Institutionalization in New Democracies". Party Politics, 8(1): 5-29.

Ruiz, Leticia and Otero, Patricia (2013). Indicadores de partidos y sistemas de partidos. Madrid: CIS.

Sani, Giacomo and Sartori, Giovani (1983). "Polarization, Fragmentation and Competition in Western Democracies". In: Daalder, H. and Mair, P. (eds.). Western European Party Systems. Beverly Hills, California/London: Sage.
Sartori, Giovanni (1976). Parties and Party Systems: A Framework for Analysis. Cambridge: Cambridge University Press.

Siaroff, Alan (2000). Comparative European Party Systems: An Analysis of Parliamentary Elections Since 1945. Oxford: Taylor and Francis.

Siaroff, Alan (2003). "Two-And-A-Half-Party Systems and the Comparative Role of the 'Half'". Party Politics, 9(3): 267-90.

Siaroff, Alan (2006). "A Typology of Contemporary Party Systems". Paper presented at the XX International Political Science Association World Congress. Fukuoka, Japan, 9th-13th July 2006. Available at: http://paperroom.ipsa.org/papers/ paper_5213.pdf, access May 8, 2018.

Sigelman, Lee and Yough, Sing (1978). "Left-Right Polarization in National Party Systems: A CrossNational Analysis". Comparative Political Studies, 11(3): 355-379.

Stoll, Heather (2008). "Social Cleavages and the Number of Parties: How the Measures you Choose Affect the Answers you Get”. Comparative Political Studies, 41: 1439-1465.

Storm, Lise (2017). "Parties and Party System Change". In: Szmolka, I. (ed.). Political Change in the Middle East and North Africa: After the Arab Spring. Edinburgh: Edinburgh University Press.

Szmolka, Inmaculada (2010). "Los regímenes políticos híbridos: democracias y autoritarismos con adjetivos. Su conceptualización, categorización y operacionalización dentro de la tipología de regímenes políticos". Revista de Estudios Políticos, 147: 103-135.

Szmolka, Inmaculada (2011). "Democracias y autoritarismos con adjetivos: la clasificación de los países árabes dentro de una tipología general de regímenes políticos". Revista Española de Ciencia Política, 26: 11-62.

Szmolka, Inmaculada (2017). "Successful and Failed Transitions to Democracy”. In: Szmolka, I. (ed.). Political Change in the Middle East and North Africa: After the Arab Spring. Edinburgh: Edinburgh University Press.

Szmolka, Inmaculada (2018). "A Real Electoral Duel between the PJD and the PAM? Analysing Constituency-level Competitiveness in the 2016 Moroccan Elections". British Journal of Middle Eastern Studies. 
Taylor, Michael and Herman, Valentine (1971). "Party Systems and Government Stability". American Political Science Review, 65(1): 28-37.

Walle, Nicolas van de and Butler, Kimberly (1999). "Political Parties and Party Systems in Africa's Illiberal Democracies". Cambridge Review of International Studies, 13(1): 14-28.

Ware, Alan (1996). Partidos políticos y sistemas de partidos. Madrid: Istmo.

Wildford, Alan (2017). "Polarization, Number of Parties, and Voter Turnout: Explaining Turnout in 26

RECEPTION: December 19, 2018

REVIEW: March 4, 2019

ACCEPTANCE: March 29, 2019
OECD Countries". Social Science Quarterly, 98(5): 1391-1405.

Wolinetz, Steven (2004). "Classifying Party Systems: Where Have All the Typologies Gone?". Annual Meeting of the Canadian Political Science Association. Winnipeg, Manitoba.

Wolinetz, Steven (2006). "Party Systems and Party System Types". In: Katz, R. and Crotty, W. (eds.). Handbook on Political Parties. London: Sage. 